\title{
Global Detection of Complex Copying Relationships Between Sources
}

\author{
Xin Luna Dong \\ AT\&T Labs-Research \\ lunadong@research.att.com \\ Yifan $\mathrm{Hu}$ \\ AT\&T Labs-Research \\ yifanhu@research.att.com
}

\author{
Laure Berti-Equille \\ Université de Rennes 1 \\ berti@irisa.fr \\ Divesh Srivastava \\ AT\&T Labs-Research \\ divesh@research.att.com
}

\begin{abstract}
Web technologies have enabled data sharing between sources but also simplified copying (and often publishing without proper attribution). The copying relationships can be complex: some sources copy from multiple sources on different subsets of data; some co-copy from the same source, and some transitively copy from another. Understanding such copying relationships is desirable both for business purposes and for improving many key components in data integration, such as resolving conflicts across various sources, reconciling distinct references to the same real-world entity, and efficiently answering queries over multiple sources. Recent works have studied how to detect copying between a pair of sources, but the techniques can fall short in the presence of complex copying relationships.

In this paper we describe techniques that discover global copying relationships between a set of structured sources. Towards this goal we make two contributions. First, we propose a global detection algorithm that identifies co-copying and transitive copying, returning only source pairs with direct copying. Second, global detection requires accurate decisions on copying direction; we significantly improve over previous techniques on this by considering various types of evidence for copying and correlation of copying on different data items. Experimental results on real-world data and synthetic data show high effectiveness and efficiency of our techniques.
\end{abstract}

\section{INTRODUCTION}

Web technologies have enabled data sources to publish and share their data, but also made it easy for sources to copy from each other (and often publish without proper attribution). The copying relationships can be complex: some sources act as data hubs and aggregate data from multiple sources; some provide only a small set of data independently, copying the rest of the data from their "friend" sources, who may also copy from others; some sources are well known and widely copied by many other sources.

Understanding the copying relationship between sources and the data flow has many benefits [1]. First, data are valuable and many

\footnotetext{
* Mobility research program supported by the European Commission (Grant FP6-MOIF-CT-2006-041000).

Permission to make digital or hard copies of all or part of this work for personal or classroom use is granted without fee provided that copies are not made or distributed for profit or commercial advantage and that copies bear this notice and the full citation on the first page. To copy otherwise, to republish, to post on servers or to redistribute to lists, requires prior specific permission and/or a fee. Articles from this volume were presented at The 36th International Conference on Very Large Data Bases, September 13-17, 2010, Singapore.

Proceedings of the VLDB Endowment, Vol. 3, No. 1

Copyright 2010 VLDB Endowment 2150-8097/10/09... \$ 10.00.
}

data providers have put a lot of money and effort in collecting and cleaning their data, so they may want to understand such relationships for business purposes (and possibly protect their own rights). Second, in data integration, considering the copying relationship can help improve truth discovery, entity resolution, schema mapping, and further optimize query answering over multiple sources. Third, identifying provenance of data can be critical for in-depth data analysis and for the study of dissemination of information. Finally, independence of sources can form an important criteria in source (user) recommendation. This paper aims at discovering complex copying relationships between a set of sources, illustrated in the following example.

EXAMPLE 1.1. We consider a data set extracted from AbeBooks. com; it includes 1263 CS books and 877 online bookstores (sources) ${ }^{1}$. Our copying-detection model (explained in the paper) predicted that between 465 pairs of sources the probability of copying is above 5 (visualized in Appendix Fig. 10, generated by GMap [9]).

First, we can cluster the bookstores by the copying relationship (as GMap does) and obtain some interesting clusters. For example, cluster Departmentstoria ${ }^{2}$ includes many big department bookstores, such as A1Books.com, Quartermelon.com, and Powell's Books; cluster Textbookistan includes many textbook stores such as www.textbooksrus.com, LGTextbooks, and brandnewtextbooks.

Second, copying relationships can be complex. Some sources (e.g., Deepak Sachdeva) seem to copy from multiple sources. Some sources (e.g., Browns Books) seem to be copied by multiple sources (and those co-copiers often do not copy from each other). Some sources seem to transitively copy from other sources; for example, Gunars Store or Gunter Koppon (one of them is a copier of the other, but the direction is unclear) transitively copies from WorldOfBooks via Books Down Under. We found that a source can copy from up to 17 sources and be copied by up to 9 sources; and there are transitive paths (where the last source indeed copies data provided by the first) of length up to 9.

Discovering copying relationships between structured sources has been studied recently in [6] for static data and in [7] for dynamic data (with updates). In particular, [6] makes pairwise decisions based on common mistakes made by the sources, and [7] considers in addition similarity of update patterns. Such techniques can detect source dependence and improve truth discovery, but may generate inaccurate copying relationships in the presence of complex copying. In particular, they have the following limitations.

\footnotetext{
${ }^{1}$ We thank the authors of [13] for providing us the data.

${ }^{2}$ We named the clusters manually.
} 
First and most importantly, these techniques consider every pair of sources in isolation of other sources and make local decisions; as a result, they cannot distinguish co-copying, transitive copying, and direct copying from multiple sources. Second, they neglect possible correlations on copying of data items; for example, a source that copies the name of a book tends to also copy its author list. Third, they view common mistakes as important evidence of copying but neglect other kinds of evidence such as whether the data are formatted in the same way, and whether two sources provide similar sets of real-world objects. Experimental results show that the second and the third limitations often lead to wrong copying directions, which in turn can lead to wrong choices among co-copying, transitive copying, and multi-source copying.

This paper proposes techniques for global copying detection on static data and these techniques can be extended for dynamic data following the ideas in [7]. Our detection proceeds in two steps: the first step locally decides possibility of copying and copying direction between each pair of sources, and the second step globally identifies co-copying and transitive copying.

This paper makes three contributions. First, for making more accurate decisions on the copying direction, critical for global detection, we enhance the previous model by gleaning more evidence such as completeness and formatting of data (Sec.3), and considering correlated copying on data items (Sec.4). Second, as a key to global detection, we introduce the techniques for discovering cocopying and transitive copying, and distinguish them from a source indeed copying from multiple sources (Sec.5). Third, we experimented on both real-world data and synthetic data, showing effectiveness and efficiency of our techniques (Sec.6 and Appendix E).

\section{OVERVIEW}

This section defines the problem we solve and describes how we profile characteristics of data.

\subsection{Problem definition}

Consider a set of real-world objects in the same domain, denoted by $\mathcal{O}$. Each object is described by a set of attributes $\mathcal{A}$, among which we assume one uniquely identifies the objects (key) ${ }^{3}$; we call an attribute $A \in \mathcal{A}$ of an object $O \in \mathcal{O}$ a data item and denote it by $O . A$. An attribute value can be atomic (e.g., string, numeric value), or a set or list of atomic values (e.g., a list of authors, a set of phone numbers), which we consider as a whole. We assume for each non-key attribute an object has a true value that reflects the reality, and many wrong values ${ }^{4}$, but for the key attribute there cannot be any wrong value (we assume entity resolution is already performed using known techniques [10]). We assume as input, we know the probability of each non-key value $v$ being true, denoted by $P(v)$ (we can compute such probabilities according to [6]).

Consider a set of sources, denoted by $\mathcal{S}$, each describing a subset of objects in $\mathcal{O}$. For each object, each source can provide values for a subset of attributes in $\mathcal{A}$ and we assume a key value must be provided. Different sources may format the same value differently (e.g., "John Smith" and "SMITH, John"); such formatting differences should be easily detectable according to some standardization or normalization rules ${ }^{5}$. For each attribute there is a set of formatting elements (e.g., for an author list, the elements can be

\footnotetext{
${ }^{3}$ It is easy to extend our techniques for the case with multi-attribute keys or the case where some attributes apply to only a subset of objects.

${ }^{4}$ Some wrong values are partially correct (e.g., misspellings and partial lists) and we can handle this case by considering value similarity as in [8].

${ }^{5}$ A standardization is not necessary; even if it is performed, the formatting information should not be discarded as it helps in copying detection.
}

Table 1: Sources in the motivating example.

\begin{tabular}{|c|c|l|c|}
\hline & ISBN & name & authors \\
\hline \multirow{3}{*}{$S_{1}$} & 1 & IPV6: Theory, Protocol, and Practice & Loshin, Peter \\
\cline { 2 - 4 } & 2 & $\begin{array}{l}\text { Web Usability: A User-Centered } \\
\text { Design Approach }\end{array}$ & Lazar, Jonathan \\
\hline \multirow{2}{*}{$S_{2}$} & 1 & IPV6 & - \\
\cline { 2 - 4 } & 2 & Web Usability & Jonathan Lazar \\
\hline \multirow{2}{*}{$S_{3}$} & 1 & IPV6: Theory, Protocol, and Practice & Loshin, Peter \\
\cline { 2 - 4 } & 2 & Web Usability & Lonathan Lazar \\
\hline \multirow{2}{*}{$S_{4}$} & 1 & IPV6: Theory, Protocol, and Practice & Lazar \\
\cline { 2 - 4 } & 2 & Web Usability & \\
\hline
\end{tabular}
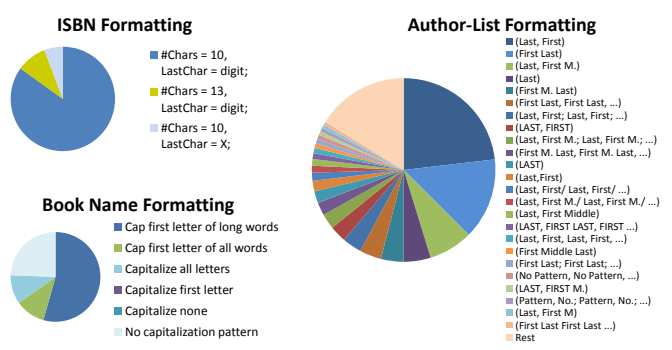

Figure 1: Formatting of attributes in the AbeBooks data set.

list completeness, name completeness, name component ordering, capitalization, etc.), each with a set of options (e.g., options for list completeness can be full author list, only first author, etc.). A formatting pattern is a combination of options of the formatting elements; such patterns can be pre-defined by observing the data. Note that some formatting patterns may contain less information than others (e.g., only first author vs. full author list) and thus they form a partial order.

EXAMPLE 2.1. Consider the four sources in Tbl.1, each providing data on the same two books. A book is described by its ISBN (the key), name, and authors ( $S_{2}$ does not provide authors for book 1). The sources may provide incorrect values (marked in italic font); e.g., $S_{2}$ provides wrong (or partially correct) values for the name of both books. They may also provide the same value but format them differently; e.g., $S_{1}$ and $S_{2}$ provide the same authors for book 2 but in different formats.

Fig.1 shows the variety of observed formatting patterns on each attribute in the AbeBooks data set. We observe much higher variety on authors than on ISBN and name.

Among the sources, some are independent and provide data independently, and some are copiers and copy all or a portion of data from other sources. A copier may verify some values and modify them when appropriate; we consider such values as independently provided, as they reflect independent observation of the real world by the copier. A copier may also reformat some copied values; we consider such values still as copied if the new format contains equal or less information (e.g., copying only the first author), and as independent if the new format contains more information (e.g., add more authors). Note that there is another kind of "dependence" between sources-negative correlation (e.g., data items provided by $S_{1}$ and $S_{2}$ are complementary, or $S_{1}$ chooses to provide different values or use different formats from $S_{2}$ ); in such cases, we consider neither source as a copier.

EXAMPLE 2.2. Continue with the motivating example. $S_{1}$ and $S_{2}$ are independent; $S_{3}$ copies the first book from $S_{1}$ and the second one from $S_{2} ; S_{4}$ copies from $S_{3}$ but has reformatted the values of authors and provides only their last names.

This paper aims to solve the following problem. 
Problem StATEMENT 1. Given a set of objects $\mathcal{O}$ and a set of sources $\mathcal{S}$, for each pair of sources $S, S^{\prime} \in \mathcal{S}$, decide the probability of $S$ directly copying from $S^{\prime}$ and vice versa.

We make a closed-world assumption on $\mathcal{O}$ and $\mathcal{S}$. This assumption on $\mathcal{O}$ should not affect the results much. That on $S$, however, can lead to predicting direct copying between co-copiers of a hidden source or between a source and its transitive copier when the direct copier is hidden. We assume there is no mutual copying; that is, $S_{1}$ copies from $S_{2}$ and $S_{2}$ copies from $S_{1}$ (on different objects).

\subsection{Data and source profiling}

A source is more likely to be a copier if the probability that it provides the observed data independently is very low. Judging this would require computing the a-priori probability that a particular source provides some particular data. There are several variations in data for a particular data item, including, but not limited to, whether a value is provided, which value is provided, and in which format the value is provided. We thus profile the data by completeness, accuracy, and formatting style, respectively, and one can define other measures similarly. These profiling measures typically fall in one of the three classes: existence measure measures whether a piece of data exists (e.g., completeness); correctness measure measures correctness of data (e.g., accuracy); and distribution measure measures distribution of values, formats, etc. (e.g., formatting style).

Note that the probability that a source provides a piece of data can depend both on source-wise statistics and data-item-wise statistics; for example, $S$ is likely to provide an object $O$ if $S$ has a high completeness or $O$ is popular. Thus, we need to define each measure both for each source and for each data item.

Completeness: The object-level completeness of a source $S$, denoted by $C_{O}(S)$, measures the percentage of objects in $\mathcal{O}$ that $S$ provides. The completeness of an object $O$, denoted by $C(O)$, measures the percentage of sources in $\mathcal{S}$ that provide $O$. Similarly, we can define attribute-level completeness.

Note that in the presence of copiers, we want to avoid being biased by them when computing completeness and other measures; for example, an object may seem popular, but most of its providers just copy data for it from a common source. We may wish to consider only independent providers; e.g., we can compute $C(O)$ by

$$
C(O)=\frac{\sum_{S \in \bar{S}(O)} P(S, O)}{\sum_{S \in \bar{S}(O)} P(S, O)+|\mathcal{S}-\bar{S}(O)|},
$$

where $\bar{S}(O)$ is the set of sources that provide $O, P(S, O)$ denotes the probability that $S$ independently provides $O$ (its computation depends on the result of copying detection), and so $\sum_{S \in \bar{S}(O)} P(S, O)$ computes the "number" of independent providers for $O$ and $\mid \mathcal{S}-$ $\bar{S}(O) \mid$ counts the number of sources that do not provide $O$.

Formatting style: The formatting style of a source $S$ measures the distribution of formatting patterns used by $S$. For each formatting pattern $f$ for $A \in \mathcal{A}$, we compute its popularity, $F_{A . f}(S)$, as the percentage of objects for which $S$ uses $f$ for the value of $A$. The formatting style of an item O.A measures the distribution of formatting patterns on $O . A$ used by different sources. We compute $F_{A . f}(O . A)$ as the percentage of sources that use $f$ for the value of $O$.A among all providers of $O . A$.

Accuracy: The accuracy of a source $S$ measures correctness of its data. We adopt techniques presented in [6] and compute accuracy by $A(S)=\operatorname{Avg}_{v \in \bar{V}(S)} P(v)$, where $\bar{V}(S)$ is the set of values provided by $S$. We can easily refine this measure for each attribute. The corresponding measure for $O . A$ is the correctness of each of $O$. A's values $v$ and is captured by $P(v)$.

We next illustrate usage of these measures in copying detection.
EXAmple 2.3. Consider $S_{1}, S_{2}$ and $S_{3}$ in Table 1. "Peter Loshin" has a misspelling (the correct spelling is "Pete Loshin") and has a low value probability, so indicates dependence between $S_{1}$ and $S_{3}$. Similarly, "Web Usability" is a wrong book name and has a low value probability, so indicates dependence between $S_{2}$ and $S_{3}$. It is more likely that $S_{3}$ copies from $S_{1}$ and $S_{2}$ than the opposite direction, as $S_{3}$ keeps the format of the copied data and thus formats authors differently for the two books, so the popularity of each formatting pattern is low (50\%).

Among these measures, we note that (1) as we show later, source copying probabilities depend on all measures we have defined; (2) the copying probabilities affect item-wise measures if we compute them considering only independent sources (e.g., by Eq.(1)), but do not affect source-wise measures; (3) the item-wise measure and the source-wise measure are independent of each other for completeness and formatting styles; however, source accuracy and value probability are inter-dependent, unless value probabilities are given upfront as input. Therefore, there is inter-dependence between data-item-wise measures, source-wise measures, and copying probabilities; we compute them iteratively until convergence, as detailed in [6].

\section{A BASIC LOCAL-DETECTION MODEL}

We now present the basic model for local copying detection assuming item-wise independence; that is, whether source $S$ copies an item $O . A$ from $S^{\prime}$ is independent of whether it copies $O^{\prime} . A^{\prime}$ from $S^{\prime}, O \neq O^{\prime}$ or $A \neq A^{\prime}$. We relax this assumption in Sec.4. Consider two sources $S_{1}, S_{2} \in \mathcal{S}, S_{1} \neq S_{2}$. The key in deciding whether $S_{1}$ copies from $S_{2}\left(S_{1} \rightarrow S_{2}\right)$ is to decide if the probability of $S_{1}$ providing the observed data conditioned on it being independent of $S_{2}$ is much lower than that conditioned on it being a copier of $S_{2}$. Intuitively, the former probability will be much lower than the latter in two cases: first, when the two sources share low-completeness items, low-probability values, or low-popularity formats; second, when there is a big difference between the profile of the overlapping data and that of $S_{1}$ 's self-provided data.

Specifically, according to the item-wise independence assumption, we consider each data item and denote by $\Phi_{O . A}(S)$ the data provided by $S$ on $O . A$. We say $\Phi_{O . A}(S)=\emptyset$ if $S$ does not provide a value for $O \cdot A$, and $\Phi_{O}(S)=\emptyset$ if $S$ does not provide a value for O.key (and so not for any other attribute either). We denote the two conditional probabilities by $P\left(\Phi_{O . A}\left(S_{1}\right) \mid S_{1} \not S_{2}\right)$ (simplified hereafter as $\left.P\left(\Phi_{O . A}\left(S_{1}\right)\right)\right)$ and $P\left(\Phi_{O . A}\left(S_{1}\right) \mid S_{1} \rightarrow S_{2}\right)$ respectively. We next focus on how we compute them according to our data profiling; our methods can be easily extended when other measures are present. The complete basic model applies Bayesian analysis by accumulating evidence from all data items and we give details in Appendix A; we give proofs of the theorems in [5].

Not copying: We start with $P\left(\Phi_{O . A}\left(S_{1}\right)\right)$. Here, $S_{1}$ does not rely on $S_{2}$ and there are three cases:

1. $S_{1}$ does not provide $O . A$ and $A$ is the key. Then $S_{1}$ does not provide $O$ (i.e., $\left.\Phi_{O}\left(S_{1}\right)=\emptyset\right)$ and

$$
P\left(\Phi_{O . A}\left(S_{1}\right)\right)=1-P\left(\Phi_{O}\left(S_{1}\right) \neq \emptyset\right) .
$$

2. $S_{1}$ does not provide $O . A$ and $A$ is not the key. Then

$$
\begin{aligned}
& P\left(\Phi_{O . A}\left(S_{1}\right) \mid \Phi_{O}\left(S_{1}\right) \neq \emptyset\right)=1-P\left(\Phi_{O . A}\left(S_{1}\right) \neq \emptyset\right) \\
& P\left(\Phi_{O . A}\left(S_{1}\right) \mid \Phi_{O}\left(S_{1}\right)=\emptyset\right)=1 .
\end{aligned}
$$

3. Otherwise, suppose $S_{1}$ provides a value $v$ and formats it in pattern $f$. Then,

$$
\begin{aligned}
P\left(\Phi_{O . A}\left(S_{1}\right)\right)= & P\left(\Phi_{O . A}\left(S_{1}\right) \neq \emptyset\right) \cdot P\left(\operatorname{value}\left(\Phi_{O . A}\left(S_{1}\right)\right)=v\right) \\
& \cdot P\left(\text { format }\left(\Phi_{O . A}\left(S_{1}\right)\right)=f\right) .
\end{aligned}
$$


Computation of $P\left(\Phi_{O}\left(S_{1}\right) \neq \emptyset\right)$ depends on the completeness of $S_{1}$ and $O$ (similar for $P\left(\Phi_{O . A}\left(S_{1}\right) \neq \emptyset\right)$ ), that of $P\left(\operatorname{value}\left(\Phi_{O . A}\left(S_{1}\right)\right)\right.$ $=v$ ) depends on $S_{1}$ 's accuracy and $v$ 's correctness, and that of $P\left(\right.$ format $\left.\left(\Phi_{O . A}\left(S_{1}\right)\right)=f\right)$ depends on the formatting styles of $S$ and $O$.A. We now present how we compute $P\left(\Phi_{O}\left(S_{1}\right) \neq \emptyset\right)$ and give details for the rest of the probabilities in Appendix A.

Intuitively, a source $S$ provides $|\mathcal{O}| C_{O}(S)$ objects, so the probabilities for providing each object should sum up to $|\mathcal{O}| C_{O}(S)$; similarly, the probabilities of each source providing $O$ should sum up to $|\mathcal{S}| C(O)$. We thus shall solve the following equations:

$$
\begin{aligned}
& \forall S \in \mathcal{S}, \sum_{O \in \mathcal{O}} P\left(\Phi_{O}(S) \neq \emptyset\right)=|\mathcal{O}| C_{O}(S) ; \\
& \forall O \in \mathcal{O}, \sum_{S \in \mathcal{S}} P\left(\Phi_{O}(S) \neq \emptyset\right)=|\mathcal{S}| C(O) .
\end{aligned}
$$

There are $|\mathcal{S}| \cdot|\mathcal{O}|$ variables but only $|\mathcal{S}|+|\mathcal{O}|$ equations, so an infinite number of solutions. We choose the one with the maximum entropy [4], so has the least bias. We can prove that in most cases ${ }^{6}$ such a solution is obtained when we assume the probability that $S$ provides each object $O$ is proportional to $C(O)$; thus,

$$
P\left(\Phi_{O}\left(S_{1}\right) \neq \emptyset\right)=\frac{|\mathcal{O}| C_{O}\left(S_{1}\right) C(O)}{\sum_{O_{0} \in \mathcal{O}} C\left(O_{0}\right)}=\frac{|\mathcal{S}| C(O) C_{O}\left(S_{1}\right)}{\sum_{S_{0} \in \mathcal{S}} C_{O}\left(S_{0}\right)} .
$$

Copying: We next compute $P\left(\Phi_{\text {O.A }}\left(S_{1}\right) \mid S_{1} \rightarrow S_{2}\right)$. Note that even if $S_{1}$ copies from $S_{2}, S_{1}$ may or may not copy a particular data item. We call the probability of copying a particular item the selectivity, and denote it by $s$. Even when $S_{1}$ copies, it can then choose to keep the original format, or to reformat it; we assume the probability of the former is $k$. We discuss how to set $s$ and $k$ in Appendix B. The computation requires comparing values and formats provided by $S_{1}$ and $S_{2}$; there are four possible cases.

1. One of $S_{1}$ and $S_{2}$ does not provide O.A. We do not penalize providing a value that the other source does not provide or vice versa (common for a copier), so

$$
P\left(\Phi_{O . A}\left(S_{1}\right) \mid S_{1} \rightarrow S_{2}\right)=P^{c}\left(\Phi_{O . A}\left(S_{1}\right)\right) .
$$

For the probability that a copier independently provides a piece of data, we mark by ${ }^{c}$ and explain in more detail shortly.

2. $S_{1}$ and $S_{2}$ provide different values on $O . A$ or $S_{1}$ uses a format with richer information. Then, $S_{1}$ does not copy:

$$
P\left(\Phi_{O . A}\left(S_{1}\right) \mid S_{1} \rightarrow S_{2}\right)=(1-s) P^{c}\left(\Phi_{O . A}\left(S_{1}\right)\right) .
$$

3. $S_{1}$ provides the same value as $S_{2}$ but in a different format $f^{\prime}\left(f^{\prime}\right.$ contains no richer information than that of $\left.S_{2}\right)$. Then, $S_{1}$ might copy (w. probability $s$ ) but reformat:

$$
\begin{aligned}
& P\left(\Phi_{O . A}\left(S_{1}\right) \mid S_{1} \rightarrow S_{2}\right)=(1-s) P^{c}\left(\Phi_{O . A}\left(S_{1}\right)\right) \\
+ & s(1-k) P^{c}\left(\operatorname{format}\left(\Phi_{O . A}\left(S_{1}\right)\right)=f^{\prime}\right) .
\end{aligned}
$$

4. $S_{1}$ provides the same value in the same format $f$. Then, $S_{1}$ might copy (w. probability $s$ ) and might follow the original format (w. probability $k$ ):

$$
\begin{aligned}
& P\left(\Phi_{O . A}\left(S_{1}\right) \mid S_{1} \rightarrow S_{2}\right)=(1-s) P^{c}\left(\Phi_{O . A}\left(S_{1}\right)\right) \\
+ & s\left(k+(1-k) P^{c}\left(\text { format }\left(\Phi_{O . A}\left(S_{1}\right)\right)=f\right)\right) .
\end{aligned}
$$

We note that we use $P^{c}\left(\Phi_{O . A}\left(S_{1}\right)\right)$ instead of $P\left(\Phi_{O . A}\left(S_{1}\right)\right)$ under condition of copying. We compute $P^{c}\left(\Phi_{O . A}\left(S_{1}\right)\right)$ in the same way as $P\left(\Phi_{O . A}\left(S_{1}\right)\right)$, except that we use "independent" measures computed only on $S_{1}$ 's data that are not copied. As we often do not know in advance which data are copied, and such computation needs to be performed for every pair of sources so needs to

\footnotetext{
${ }^{6}$ The only exception happens when $\frac{|\mathcal{O}| C_{O}\left(S_{1}\right) C(O)}{\sum_{O_{0} \in \mathcal{O}} C\left(O_{0}\right)}>1$ for some $S_{1}$ and $O$; in this case, we can estimate by setting $P\left(\Phi_{O}\left(S_{1}\right) \neq \emptyset\right)=1$ for such $S_{1}$ and $O$, and compute by Eq.(8) for other sources and objects .
}

Table 2: Ex. 3.2 and 4.1. Each table describes data provided by two sources on 5 objects, each with 5 attributes ( $K$ is the key). " $S$ " in the table indicates that the two sources provide the same value in the same format, and " $D$ " indicates that they provide different values. Copying seems more likely for (b) than for (a).

\begin{tabular}{|c|ccccc|}
\hline & $K$ & $A_{1}$ & $A_{2}$ & $A_{3}$ & $A_{4}$ \\
\hline$O_{1}$ & $\mathrm{~S}$ & $\mathrm{~S}$ & $\mathrm{~S}$ & $\mathrm{D}$ & $\mathrm{D}$ \\
$O_{2}$ & $\mathrm{~S}$ & $\mathrm{D}$ & $\mathrm{S}$ & $\mathrm{S}$ & $\mathrm{D}$ \\
$O_{3}$ & $\mathrm{~S}$ & $\mathrm{~S}$ & $\mathrm{D}$ & $\mathrm{S}$ & $\mathrm{D}$ \\
$O_{4}$ & $\mathrm{~S}$ & $\mathrm{~S}$ & $\mathrm{~S}$ & $\mathrm{D}$ & $\mathrm{S}$ \\
$O_{5}$ & $\mathrm{~S}$ & $\mathrm{D}$ & $\mathrm{S}$ & $\mathrm{S}$ & $\mathrm{S}$ \\
\hline \multicolumn{7}{|c}{ (a) }
\end{tabular}

\begin{tabular}{|c|ccccc|}
\hline & $K$ & $A_{1}$ & $A_{2}$ & $A_{3}$ & $A_{4}$ \\
\hline$O_{1}$ & $\mathrm{~S}$ & $\mathrm{~S}$ & $\mathrm{~S}$ & $\mathrm{~S}$ & $\mathrm{~S}$ \\
$O_{2}$ & $\mathrm{~S}$ & $\mathrm{~S}$ & $\mathrm{~S}$ & $\mathrm{~S}$ & $\mathrm{~S}$ \\
$O_{3}$ & $\mathrm{~S}$ & $\mathrm{~S}$ & $\mathrm{~S}$ & $\mathrm{~S}$ & $\mathrm{~S}$ \\
$O_{4}$ & $\mathrm{~S}$ & $\mathrm{D}$ & $\mathrm{D}$ & $\mathrm{D}$ & $\mathrm{D}$ \\
$O_{5}$ & $\mathrm{~S}$ & $\mathrm{D}$ & $\mathrm{D}$ & $\mathrm{D}$ & $\mathrm{D}$ \\
\hline \multicolumn{7}{|c}{ (b) }
\end{tabular}

be very fast, we estimate these measures. As an example, we estimate $S_{1}$ 's "independent" object-level completeness w.r.t. $S_{2}$ as its completeness on objects not provided by $S_{2}$ :

$$
C_{O}\left(S_{1} \mid \neg S_{2}\right)=\frac{\left|\bar{O}\left(S_{1}\right)\right|-\left|\bar{O}\left(S_{1}\right) \cap \bar{O}\left(S_{2}\right)\right|}{|\mathcal{O}|-\left|\bar{O}\left(S_{2}\right)\right|},
$$

where $\bar{O}(S)$ denotes the set of objects provided by $S$.

Discussion: The following result lists positive evidence for copying, conforming to our intuitions.

Proposition 3.1. Given sources $S_{1}$ and $S_{2}$ and data item $O . A$, in the following cases $O$.A forms positive evidence for $S_{1} \rightarrow S_{2}$.

- $S_{1}$ provides the same value in the same format as $S_{2}$ on $O . A$, and $P\left(\Phi_{O . A}\left(S_{1}\right)\right)<s k$;

- $S_{1}$ provides the same value but uses a different format $f^{\prime}$, and $P\left(\Phi_{O . A}\left(S_{1}\right)\right)<s(1-k) P^{c}\left(\right.$ format $\left.\left(\Phi_{O . A}\left(S_{1}\right)\right)=f^{\prime}\right)$;

- $P^{c}\left(\Phi_{O . A}\left(S_{1}\right)\right)>P\left(\Phi_{O . A}\left(S_{1}\right)\right)$ and $S_{2}$ does not provide $O . A$;

- $(1-s) P^{c}\left(\Phi_{O . A}\left(S_{1}\right)\right)>P\left(\Phi_{O . A}\left(S_{1}\right)\right)$.

EXAMPLE 3.2. Consider sources $S_{1}$ and $S_{2}$, each providing 5 objects and 5 attributes for each object (shown in Tbl. 2(a)). Assume $P\left(\Phi_{O . K}\left(S_{1}\right)\right)=P^{c}\left(\Phi_{O . K}\left(S_{1}\right)\right)=.9, P\left(\Phi_{O . A_{i}}\left(S_{1}\right)\right)=$ $P^{c}\left(\Phi_{O . A_{i}}\left(S_{1}\right)\right)=.5, i \in[1,4]$, and $P^{c}\left(\operatorname{format}\left(\Phi_{O . A}\left(S_{1}\right)\right)\right)=$ .8 for each attribute $A$. We set $s=.6, k=.5$.

We first compute $P\left(\Phi\left(S_{1}\right) \mid S_{1} \rightarrow S_{2}\right)\left(\Phi\left(S_{1}\right)\right.$ denotes observation of $S_{1}$ 's data). There are 5 key items on which the two sources provide the same value in the same format; the probability is (1$.6) * .9+.6 *(.5+(1-.5) * .8)=.4 * .9+.6 * .9=.9($ Eq.(12)). There are 12 non-key items on which the two sources provide the same value in the same format; the probability is $.4 * .5+.6 * .9=.74$ (Eq.(12)). Finally, there are 8 items on which the two sources provide different values and the probability is $.4 * .5=.2$ (Eq.(10)). So $P\left(\Phi\left(S_{1}\right) \mid S_{1} \rightarrow S_{2}\right)=.9^{5} * .74^{12} * .2^{8}$.

On the other hand, it is obvious that $P\left(\Phi\left(S_{1}\right) \mid S_{1} \nrightarrow S_{2}\right)=$ $.9^{5} * .5^{20}$. So $\frac{P\left(\Phi\left(S_{1}\right) \mid S_{1} \rightarrow S_{2}\right)}{P\left(\Phi\left(S_{1}\right) \mid S_{1} \nrightarrow S_{2}\right)}=.07$ and $S_{1}$ is unlikely to be a copier of $S_{2}$. This is reasonable because $S_{1}$ provides a lot of values differently from $S_{2}$, and for the values they share, $S_{1}$ has a relatively high probability to provide them by itself.

Comparison with [6]: There are three differences between our basic model and the model presented in [6].

1. The basic model allows the flexibility of plugging in evidence of various types, including completeness and formatting of data in addition to correctness of data.

2. In addition to source-wise measures, we consider also itemwise measures when computing $P\left(\Phi_{O . A}(S)\right)$.

3. Instead of using $P\left(\Phi_{O . A}\left(S_{1}\right)\right)$, we use $P^{c}\left(\Phi_{O . A}\left(S_{1}\right)\right)$ under condition $S_{1} \rightarrow S_{2}$.

Note that difference 3 is a correction of the previous model; however, our experiments show that it does not necessarily improve the results when we consider only data correctness. Finally, none of the techniques in Sec.4-5 is included in [6]. 


\section{CORRELATED COPYING}

The basic model assumes item-wise independence, which seldom holds in reality. For example, the copier may compose a SQL query and copy all returned objects; when it copies an object, it often copies all provided attributes or the attributes in its own schema. This section discusses object copying (the latter); similar techniques can be applied for query-driven copying (the former).

One can imagine that a copier often copies in one of two modes: 1) it copies a subset of objects on a subset of attributes, called per-object copying; 2) it copies on a subset of attributes for a set of independently provided objects (or objects copied from other sources), called per-attribute copying. The difference is whether the copier also copies the key values or not. Thus, when we compute $P\left(\Phi_{O . A}\left(S_{1}\right) \mid S_{1} \rightarrow S_{2}\right)$, we need to consider whether $S_{1}$ copies on $O$ or only on $A$ and treat them differently.

Specifically, we denote by $S_{1} \stackrel{O}{\longrightarrow} S_{2}$ that $S_{1}$ copies $O$ from $S_{2}$, by $S_{1} \stackrel{\text { O.A }}{\longrightarrow} S_{2}$ that $S_{1}$ copies $O . A$ from $S_{2}$, and simplify $P\left(S_{1} \stackrel{O}{\longrightarrow} S_{2}\right.$ $\left.S_{1} \rightarrow S_{2}\right)$ as $s(O)(s(O)$ can be viewed as the selectivity on $O$ for $S_{1} \rightarrow S_{2}$, but we omit $S_{1} \rightarrow S_{2}$ for simplicity). Then, we have

$$
\begin{aligned}
& P\left(\Phi_{O . A}\left(S_{1}\right) \mid S_{1} \rightarrow S_{2}\right)=s(O) P\left(\Phi_{O . A}\left(S_{1}\right) \mid S_{1} \stackrel{O}{\longrightarrow} S_{2}\right) \\
+ & \left.(1-s(O)) P\left(\Phi_{O . A}\left(S_{1}\right) \mid S_{1} \stackrel{O}{\longrightarrow} S_{2}, S_{1} \rightarrow S_{2}\right)\right) .
\end{aligned}
$$

The computation of $P\left(\Phi_{O . A}\left(S_{1}\right) \mid S_{1} \stackrel{O}{\longrightarrow} S_{2}\right)$ and $P\left(\Phi_{O . A}\left(S_{1}\right) \mid\right.$ $\left.S_{1} \stackrel{O}{\rightarrow} S_{2}, S_{1} \rightarrow S_{2}\right)$ is the same as $P\left(\Phi_{O . A}\left(S_{1}\right) \mid S_{1} \rightarrow S_{2}\right)$, except that we shall set the selectivity differently. We denote the selectivity for an attribute $A \in \mathcal{A}$ of a copied object by $s_{A}$ and that of an uncopied object (per-attribute copying) by $s_{A}^{\rightarrow}$. According to

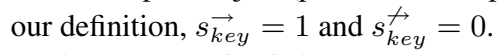

The next question is how to compute $s(O)$ depending on our observation of data provided on $O$. Let $s_{o b j}$ be the a-priori probability that a copier copies an object. Then, by Bayesian analysis,

$$
\begin{aligned}
& s(O)=P\left(S_{1} \stackrel{O}{\longrightarrow} S_{2} \mid \Phi_{O}\left(S_{1}\right), S_{1} \rightarrow S_{2}\right) \\
= & \frac{s_{o b j} P\left(\Phi_{O}\left(S_{1}\right) \mid S_{1} \stackrel{O}{\longrightarrow} S_{2}\right)}{s_{o b j} P\left(\Phi_{O}\left(S_{1}\right) \mid S_{1} \stackrel{O}{\longrightarrow} S_{2}\right)+\left(1-s_{o b j}\right) P\left(\Phi_{O} \mid S_{1} \stackrel{O}{\rightarrow} S_{2}, S_{1} \rightarrow S_{2}\right)}
\end{aligned}
$$

We can compute $P\left(\Phi_{O}\left(S_{1}\right) \mid S_{1} \stackrel{O}{\longrightarrow} S_{2}\right)$ from $P\left(\Phi_{O . A}\left(S_{1}\right) \mid S_{1} \stackrel{O}{\longrightarrow} S_{2}\right)$, $A \in \mathcal{A}$, assuming all attributes are independent (we can relax this assumption by further grouping the attributes).

One big challenge for applying the enhanced model is parameter setting. The enhanced model introduces new parameters, $s_{o b j}, s_{A}$, and $s_{A}^{\nrightarrow}$; they are essentially conditional probabilities and can vary from source pair to source pair. Setting them appropriately is important in achieving accurate results. We set them for each direction of each pair of sources in two steps: first, we initialize them empirically according to the data; second, we adjust them later according to copying-detection results and re-apply our model accordingly. Appendix B gives the details.

EXAMPLE 4.1. Continue with Ex.3.2 and now consider $S_{1}$ and $S_{2}$ in Tbl. 2(b). With the same calculations as in Ex.3.2, $S_{1}$ appears unlikely to be a copier of $S_{2}$. Now consider per-object copying and we set $s_{o b j}=.6$ and $s_{A}=.9, s_{A}^{\rightarrow}=.1$ for each attribute.

For each non-key item in $\left\{\mathrm{O}_{1}, \mathrm{O}_{2}, \mathrm{O}_{3}\right\}$, if $\mathrm{S}_{1}$ copies the object, the probability that it provides the data is $.1 * .5+.9 * .9=.86$ (Eq.(12)); otherwise, if $S_{1}$ copies from $S_{2}$ but not on the object, the probability becomes $.9 * .5+.1 * .9=.54$. For each non-key item in $\left\{O_{4}, O_{5}\right\}$, if $S_{1}$ copies the object, the probability is $.1 * .5=.05$ (Eq.(10)); otherwise, the probability is $.9 * .5=.45$. Finally, for each key attribute, the probability that $S_{1}$ provides it is always.9.
Table 3: Three sets of data sources. In each one, $S_{1}$ and $S_{2}$ share 50 values, $S_{1}$ and $S_{3}$ share 50 values, and $S_{2}$ and $S_{3}$ share 30 values.

\begin{tabular}{|c|c|c|c|}
\hline Src & $\mathcal{D}_{1}$ (Multi-copy) & $\mathcal{D}_{2}$ (Co-copy) & $\mathcal{D}_{3}$ (Transitive) \\
\hline$S_{1}$ & \multicolumn{3}{|c|}{$v_{1} \sim v_{100}$, where $v_{81} \sim v_{100}$ are popular values } \\
\hline$S_{2}$ & $v_{1} \sim v_{50}, v_{101} \sim v_{130}$ & $v_{1} \sim v_{50}$ & $v_{1} \sim v_{50}$ \\
\hline$S_{3}$ & $v_{51} \sim v_{130}$ & $v_{21} \sim v_{70}$ & $v_{21} \sim v_{50}, v_{81} \sim v_{100}$ \\
\hline
\end{tabular}

Accordingly, the probability that $\mathrm{S}_{1}$ copies $\mathrm{O}_{1}, \mathrm{O}_{2}$ or $\mathrm{O}_{3}$ is $\frac{.6 *\left(.9 * .86^{4}\right)}{.6 *\left(.9 * .86^{4}\right)+.4 *\left(.9 * .54^{4}\right)}=.9(E q .(15))$. The probability that it provides each non-key value is thus $.9 * .86+.1 * .54=.83$ (Eq.(14)). Similarly, the probability that $\mathrm{S}_{1}$ copies $\mathrm{O}_{4}$ or $\mathrm{O}_{5}$ is .002 and the probability that it provides each non-key value is .45 . Therefore, $\frac{P\left(\Phi\left(S_{1}\right) \mid S_{1} \rightarrow S_{2}\right)}{P\left(\Phi\left(S_{1}\right) \mid S_{1} \nrightarrow S_{2}\right)}=\frac{.9^{5} * .83^{12} * .45^{8}}{.9^{5} * .5^{20}}=188$ and $S_{1}$ is likely to be a copier of $S_{2}$.

To compare, for Tbl.2(a), considering per-object copying obtains a ratio of 1.37 and still does not strongly imply copying.

\section{GLOBAL COPYING DETECTION}

Local detection aims at discovering (positive) dependence between sources; however, such dependence is not always caused by direct copying, but can also be due to co-copying or transitive copying. In the motivating example (Tbl.1), local detection may conclude with $S_{4} \rightarrow S_{1}$ and $S_{4} \rightarrow S_{2}$, although $S_{4}$ only transitively copies from $S_{1}$ and $S_{2}$. Global detection tries to fix this problem. However, identifying co-copying and transitive copying is non-trivial, as the following example illustrates.

EXAMPLE 5.1. Consider $S_{1}, S_{2}$ and $S_{3}$, where local detection decides that $S_{2} \rightarrow S_{1}, S_{3} \rightarrow S_{1}$, and $S_{3} \rightarrow S_{2}$. We need to decide if $S_{3}$ copies only from $S_{1}$ (co-copying with $S_{2}$ ), only from $S_{2}$ (transitively copying from $S_{1}$ ), or from both (multi-source copying).

One may consider finding a clue from the copying probabilities, but this often does not work when each pair of sources share a lot of values and thus have a copying probability of 1 .

One may then consider comparing the numbers of overlapping values, but this is insufficient. Consider $\mathcal{D}_{1}$ and $\mathcal{D}_{2}$ in Tbl.3. Each pair of sources share the same number of values for the two cases; however, because of the different distribution of the shared values, $\mathcal{D}_{1}$ seems more likely to have multi-source copying, whereas $\mathcal{D}_{2}$ seems more likely to have co-copying.

One may next consider comparing the sets of overlapping values, but this is still insufficient. Consider $\mathcal{D}_{2}$ and $\mathcal{D}_{3}$ in Tbl.3. Values that all of $S_{1}, S_{2}$ and $S_{3}$ share are the same $\left(v_{21} \sim v_{50}\right)$ in the two cases; however, $\mathcal{D}_{2}$ seems more likely to have a co-copying, whereas $\mathcal{D}_{3}$ seems more likely to have a transitive copying, because the rest of the 20 values shared between $S_{1}$ and $S_{3}$ in $\mathcal{D}_{3}$ are popular values and $S_{3}$ may provide them independently. Thus, we need to reason for each data item in a more principled way.

Our key intuition is that since co-copying and transitive copying can often be inferred from direct copying, we first find a set of copying relationships $\mathbf{R}$ that significantly influence the rest of the relationships, and then adjust the rest accordingly and decide if each is a direct or indirect copying (the results are denoted by $\left.P\left(S_{1} \rightarrow S_{2} \mid \mathbf{R}\right),\left(S_{1}, S_{2}\right) \notin \mathbf{R}\right)$. In this process we need to solve two problems: (1) how to select the set $\mathbf{R}$; and (2) how to compute $P\left(S_{1} \rightarrow S_{2} \mid \mathbf{R}\right)$. The first problem relies on the second, so we start from our solution for the second problem.

Computing $P\left(S_{1} \rightarrow S_{2} \mid \mathbf{R}\right)$ : As we have illustrated, we cannot derive this probability directly from the copying probabilities in $\mathbf{R}$, but have to reason for each data item if $S_{1}$ is likely to copy it from $S_{2}$ even in the presence of the copying between $S_{1}$ and other sources. Thus, when we compute $P\left(S_{1} \rightarrow S_{2} \mid \mathbf{R}\right)$, we replace 
$P\left(\Phi_{O . A}\left(S_{1}\right)\right)$ everywhere with $P\left(\Phi_{O . A}\left(S_{1}\right) \mid \mathbf{R}\right)$, the probability that $S_{1}$ independently provides the data or copies the data from other sources according to $\mathbf{R}$. We next illustrate how we compute $P\left(\Phi_{O . A}\left(S_{1}\right) \mid \mathbf{R}\right)$ using the case of $\Phi_{O . A}\left(S_{1}\right) \neq \emptyset$.

Consider the set of sources that are associated with $S_{1}$ by some copying relationship in $\mathbf{R}$. Consider two subsets: $\bar{S}_{f}(O . A)$, those providing the same value in the same format on $O . A$ as $S_{1} ; \bar{S}_{v}(O . A)$, those providing the same value in a different format. The probability that $S_{1}$ does not copy O.A from any source in $\bar{S}_{f}(O . A)$ is

$$
\begin{aligned}
P_{f}=\sum_{S \in \bar{S}_{f}(O . A)}\left(1-P\left(S_{1} \rightarrow S\right) P\left(S_{1} \stackrel{\text { O.A }}{\longrightarrow} S\right)\right. \\
\left.\cdot\left(k+(1-k) P\left(\operatorname{format}\left(\Phi_{O . A}\left(S_{1}\right)\right)=f\right)\right)\right)(16)
\end{aligned}
$$

where $P\left(S_{1} \rightarrow S\right)$ and $P\left(S_{1} \stackrel{\text { O.A }}{\rightarrow} S\right)$ are inferred from R. Similarly, we can compute the probability that $S_{1}$ does not copy O.A from any source in $\bar{S}_{v}(O . A)$ and provide the observed format, denoted by $P_{v}$. Then $S_{1}$ either provides the data by itself (with probability $\left.P_{v} P_{f}\right)$, or copies $O . A$ from $\bar{S}_{v}(O . A)$ or $\bar{S}_{f}(O . A)$, so

$$
P\left(\Phi_{O . A}\left(S_{1}\right) \mid \mathbf{R}\right)=\left(1-P_{v} P_{f}\right)+P_{v} P_{f} P\left(\Phi_{O . A}\left(S_{1}\right)\right) .
$$

EXAMPle 5.2. Continue with Ex.5.1. Consider $\mathcal{D}_{1}$ and suppose $\mathbf{R}=\left\{\left(S_{1}, S_{3}\right)\right\}$. For each $v \in\left\{v_{101}, \ldots, v_{130}\right\}, \Phi_{v}\left(S_{3}\right)=$ $\Phi_{v}\left(S_{3} \mid \mathbf{R}\right)$, so $S_{3}$ still looks like a copier of $S_{2}$.

Consider $\mathcal{D}_{2}$ and suppose $\mathbf{R}=\left\{\left(S_{1}, S_{3}\right)\right\}$. For each $v \in$ $\left\{v_{21}, \ldots, v_{50}\right\}, \Phi_{v}\left(S_{3} \mid \mathbf{R}\right)$ is much larger than $\Phi_{v}\left(S_{3}\right)$, so $S_{3}$ looks much less likely a copier of $S_{2}$.

Finally, consider $\mathcal{D}_{3}$ and suppose $\mathbf{R}=\left\{\left(S_{2}, S_{3}\right)\right\}$. For each $v \in\left\{v_{21}, \ldots, v_{50}\right\}$, again $\Phi_{v}\left(S_{3} \mid \mathbf{R}\right)$ is much larger than $\Phi_{v}\left(S_{3}\right)$; for each $v \in\left\{v_{81}, \ldots, v_{100}\right\}, \Phi_{v}\left(S_{3} \mid \mathbf{R}\right)=\Phi_{v}\left(S_{3}\right)$ but is high. Thus, $S_{3}$ looks less likely a copier of $S_{1}$.

Finding R: Finding a proper set of relationships for $\mathbf{R}$ is crucial. As an example, for $\mathcal{D}_{2}$ in Tbl.3, if we include $S_{3} \rightarrow S_{2}$ in $\mathbf{R}$, we will not be able to detect the real copying $S_{3} \rightarrow S_{1}$. We wish to include in $\mathbf{R}$ the most influential copying relationships; that is, our goal is to find the set $\mathbf{R}$ that maximizes

$$
\psi(\mathbf{R})=\sum_{\left\langle S_{1}, S_{2}\right\rangle \notin \mathbf{R}}\left(P\left(S_{1} \rightarrow S_{2}\right)-P\left(S_{1} \rightarrow S_{2} \mid \mathbf{R}\right)\right) .
$$

(We shall consider only positive influence and if $P\left(S_{1} \rightarrow S_{2}\right)-$ $P\left(S_{1} \rightarrow S_{2} \mid \mathbf{R}\right)<0$, we set it to 0 .) We can prove the NP-hardness of this problem by a reduction from the HITTING SET problem.

THEOREM 5.3. The problem of finding the most influential copying relationships $\mathbf{R}$ is NP-complete.

We now present several observations, based on which we propose a fast greedy algorithm. The first observation is that according to Eq.(17), $P\left(S_{1} \rightarrow S_{2} \mid \mathbf{R}\right)$ relies on only relationships involving $S_{1}$. Thus, we can construct $\mathbf{R}$ by finding for each source the most "influential" sources among those it may copy from.

Proposition 5.4. If we denote by $\bar{D}\left(S_{1}\right), S_{1} \in \mathcal{S}$, the sources with which $S_{1}$ has a copying relationship in $\mathbf{R}$, by $\mathbf{R}\left(S_{1}\right)$ the relationships in $\mathbf{R}$ involving $S_{1}$, and by $\Delta\left(S_{1} \rightarrow S_{2} \mid \bar{D}\left(S_{1}\right)\right)=$ $P\left(S_{1} \rightarrow S_{2}\right)-P\left(S_{1} \rightarrow S_{2} \mid \mathbf{R}\left(S_{1}\right)\right)$, we have $\psi(\mathbf{R})=\sum_{S_{1} \in \mathcal{S}}$ $\sum_{S_{2} \notin \bar{D}\left(S_{1}\right)} \Delta\left(S_{1} \rightarrow S_{2} \mid \bar{D}\left(S_{1}\right)\right)$.

The second observation reveals the relationship between the joint influence of sources in $\bar{D}\left(S_{1}\right)$ and the individual influence of each of them. Accordingly, we can simplify our algorithm by considering influence of an individual copying relationship on another.
Proposition 5.5. If we denote by $\Delta\left(S_{1} \rightarrow S_{2} \mid S\right)=P\left(S_{1} \rightarrow\right.$ $\left.S_{2}\right)-P\left(S_{1} \rightarrow S_{2} \mid\left\{S_{1} \rightarrow S\right\}\right)$, then, (1) $\Delta\left(S_{1} \rightarrow S_{2} \mid \bar{D}\left(S_{1}\right)\right) \geq$ $\Delta\left(S_{1} \rightarrow S_{2} \mid S\right)$ for each $S \in \bar{D}\left(S_{1}\right)$; and (2) $\Delta\left(S_{1} \rightarrow S_{2} \mid \bar{D}\left(S_{1}\right)\right)$ $\leq \sum_{S \in \bar{D}\left(S_{1}\right)} \Delta\left(S_{1} \rightarrow S_{2} \mid S\right)$.

Based on Proposition 5.5, we wish to greedily select relationships that have the highest accumulated influence on others. The next two observations state which relationships should be pruned (not added to $\mathbf{R}$ ). The third observation shows that we want to prune relationships that cause less accumulated changes on others than being affected by others; its proof is based on Proposition 5.5.

Proposition 5.6. For any $S \in \mathcal{S}$, if there exist $S_{1}$ and $S_{2}$, $S_{1} \neq S_{2}$, such that (1) $\Delta\left(S \rightarrow S_{1} \mid S_{2}\right)>\sum_{S_{0} \neq S, S_{1}, S_{2}} \Delta(S \rightarrow$ $\left.S_{0} \mid S_{1}\right)$, (2) $\left(S, S_{2}\right) \in \mathbf{R}$, and (3) $\mathbf{R}^{\prime}=\mathbf{R} \cup\left\{\left(S, S_{1}\right)\right\}$, then $\psi(\mathbf{R})>\psi\left(\mathbf{R}^{\prime}\right)$.

The final observation shows that we should prune a relationship if it can be significantly influenced by those already selected into $\mathbf{R}$, because it is more likely to be a co-copying or transitive copying and its influence on others will be dominated by the relationships in $\mathbf{R}$.

ObSERVATION 5.7. For any $S \in \mathcal{S}$, if there exist $S_{1}$ and $S_{2}$, $S_{1} \neq S_{2}$, such that (1) $\Delta\left(S \rightarrow S_{1} \mid\left\{S \rightarrow S_{2}\right\}\right)>.5$, (2) $\left(S, S_{2}\right) \in \mathbf{R}$, and (3) $\mathbf{R}^{\prime}=\mathbf{R} \cup\left\{\left(S, S_{1}\right)\right\}$, then typically $\psi(\mathbf{R})>$ $\psi\left(\mathbf{R}^{\prime}\right)$.

Based on these observations, for each source $S \in \mathcal{S}$ our algorithm proceeds in four steps.

1. Find all sources from which $S$ is likely to copy or copying is likely but the direction is unclear, denoted by $\bar{R}(S)$.

2. For each $S_{1}, S_{2} \in \bar{R}(S), S_{1} \neq S_{2}$, compute $\Delta\left(S \rightarrow S_{1} \mid S_{2}\right)$ and $\Delta\left(S \rightarrow S_{2} \mid S_{1}\right)$ (influence). For each $S^{\prime} \in \bar{R}(S)$, compute $\sigma\left(S^{\prime}\right)=\sum_{S_{0} \neq S, S^{\prime}} \Delta\left(S \rightarrow S_{0} \mid S^{\prime}\right)$ (influence on others) and $\Lambda\left(S^{\prime}\right)=\max _{S_{0} \neq S, S^{\prime}} \Delta\left(S \rightarrow S^{\prime} \mid S_{0}\right)$ (max influence by others).

3. Find the source $S^{\prime}$ with the highest $\sigma\left(S^{\prime}\right)$ (most influential) and remove it from $\bar{R}(S)$. If $\sigma\left(S^{\prime}\right)>\Lambda\left(S^{\prime}\right)$ (affecting others more than being affected), (1) add $\left(S, S^{\prime}\right)$ to $\mathbf{R},(2)$ for each $S_{0} \in \bar{R}(S)$, if $P\left(S \rightarrow S_{0} \mid\left\{S \rightarrow S^{\prime}\right\}\right)<.5$, remove $S_{0}$ from $\bar{R}(S)$ (pruning); (3) update $\sigma\left(S_{0}\right), S_{0} \in \bar{R}(S)$, by ignoring the removed sources.

4. Go to step 3 until $\bar{R}(S)=\emptyset$.

EXAMPLE 5.8. Consider $S_{4}$ in the motivating example (Tbl.1). Assume $\Delta\left(S_{4} \rightarrow S_{1} \mid S_{3}\right)=.8, \Delta\left(S_{4} \rightarrow S_{2} \mid S_{3}\right)=.8, \Delta\left(S_{4} \rightarrow\right.$ $\left.S_{3} \mid S_{1}\right)=.5, \Delta\left(S_{4} \rightarrow S_{3} \mid S_{2}\right)=.5 . S_{4} \rightarrow S_{3}$ has the highest accumulated influence (1.6) and is less influenced by others (.5), so we add it to $\mathbf{R}$. Since both $S_{4} \rightarrow S_{1}$ and $S_{4} \rightarrow S_{2}$ are significantly influenced by $S_{4} \rightarrow S_{3}$, we can prune them and terminate.

Appendix $\mathrm{C}$ gives the detailed algorithm GlobalDeteCtion and describes opportunities for further approximation.

Proposition 5.9. Let $m=\max _{S \in \mathcal{S}}|\bar{R}(S)|$ and $r$ be the maximum number of sources related to a source in $\mathbf{R}$. The complexity of GLOBALDETECTION is $O\left(m^{2}|\mathcal{S}||\mathcal{O}|+r|\mathcal{S}|^{2}|\mathcal{O}|\right)$.

\section{EXPERIMENTAL RESULTS}

We experimented on two real-world data sets: the weather data (described in Sec. 6.1) and the AbeBooks data (Sec. 6.2). They are very different in the size of the domain, the number of the sources, and the characteristics of the data. We have a partial golden standard for the first but no golden standard for the second; thus, we focus on the correctness of our results on the first and the efficiency 


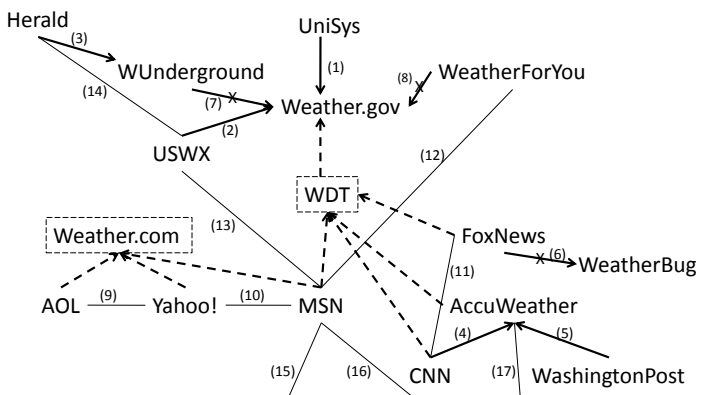

MyForecast FindLocalWeather Climaton NYTimes

Figure 5: Copying between weather sources. There are 18 sources and 2 hidden sources (in dashed box). A solid arrow represents a copying indicated by the source website (non-crossed ones are "golden" dependencies); a dashed arrow represents a copying associated w. a hidden source; and a thin line represents a "silver" dependence that we derive.

of our algorithm on the second. Together with synthetic data generated from the AbeBooks data (Appendix E), we are able to test various aspects of our models, showing their effectiveness, stability, and efficiency.

We implemented the model in [6] (ACCU), the basic model (BASIC), the correlated-copying model (LOCAL), and global detection (GlobAL). We set the parameters as described in Appendix B, with initial values $\alpha=.25$ and $k_{0}=.8$. We did not assume knowledge of correctness or popularity of values and conducted truth finding and copying detection iteratively (Sec.2). We used Java and experimented on a WindowsXP machine with $2.66 \mathrm{GHz}$ Intel CPU and 3.48GB of RAM.

\subsection{Results on the weather data}

Data: We collected weather data for 30 major USA cities from 18 websites about every 45 minutes. We consider (city, time) as the key. There are in total 33 collections in a day and thus 990 objects. We manually map the attributes and there are 28 distinct attributes. Among them, 10 are provided by at least 10 sources and 11 are provided by only 1 source; on the other hand, a source on average provides 11 attributes, while the $\max$ is 15 and the $\min$ is 3 .

This data set introduces four new challenges. First, there is hardly a true or false notion for weather-related data; thus, we need to consider popularity of provided values and we do so in a similar way as we consider formatting popularity. Second, the weather data are often updated frequently and a copier may not have copied the most up-to-date data at some time of crawling; thus, we need to be able to detect copying even when the copying percentage is not high and so setting proper parameters is critical. Third, most sources have the same object-level completeness and similar completeness for each attribute, and each source has a consistent formatting style for the same attribute (by applying some style sheet); thus, we lack evidence from completeness and formatting for direction decision. Fourth, there are two hidden sources, $W D T^{7}$ and Weather.com, that are co-copied by sources in our data set, but we cannot crawl them because of commercial or technical reasons.

Golden standard: To find copying between sources, we investigated the websites for explicitly mentioned partnerships, clients, and resources; in addition, we checked source code of the webpages for URLs and citations of other sources. We found 8 copyings between the crawled sources and 8 from the crawled sources to the hidden ones (Fig. 5). Accordingly, we created a semi-golden standard as follows. First, we manually examined every pair of sources

\footnotetext{
${ }^{7}$ WDT collects raw data from Weather.gov and applies some aggregation model, then resells the data to online media agencies.
}

Table 4: Results of various methods on the weather data.

\begin{tabular}{|c|c|c|c|c|}
\hline & ACCU & BASIC & LOCAL & GLOBAL \\
\hline Precision & .5 & 1 & .33 & .79 \\
Recall & .43 & .14 & .86 & .79 \\
F-measure & .46 & .25 & .48 & .79 \\
\hline
\end{tabular}

with investigated copying; we removed 3 of them where we observed very small commonality of data with no particular pattern. The remaining 5 are called golden dependencies. Second, for each copier of a hidden source, we tried to find its co-copiers with which it shares a large portion of common data; if such co-copiers exist, we added a dependence (with no particular direction) with the one with the largest overlap. We added 5 dependencies in this way and call them silver dependencies. Third, for each source where we cannot find any claim from the website, we manually checked if there exist sources that share a large portion of common data; if so we added a dependence (without direction) with the one with the largest overlap. There are 5 such sources and we added 4 dependencies, also called silver dependencies. In total, there are 14 copyings ( 5 golden and 9 silver) in the semi-golden standard and we list the reasons for including them in Appendix D.

Measures: Here we focus on whether our model correctly finds direct copying and defer testing correctness of copying direction to the synthetic data (Appendix E). This is because as mentioned, the weather data lack evidence from formatting for direction detection, which is shown to be critical from results on the synthetic data. Specifically, we measured precision, the proportion of identified copying that are real (maybe in the wrong direction), and recall, the proportion of real copying that are identified. F-measure is computed as $\frac{2 P R}{P+R}$, where $P$ is the precision and $R$ is the recall.

Results: GloBAL obtained both precision and recall as .79. (1) Among the 5 golden dependencies, Global finds 4 of them, 2 in the correct direction and 1 with uncertain direction. It misses Herald $\rightarrow$ WUnderground: among the 8 common non-key attributes, they highly overlap only on conditions and visibility, and the shared values are fairly common also among other sources (especially for visibility), so the accumulated positive evidence is slightly weaker than the negative evidence, even with a reasonable parameter setting. (2) Among the 9 silver dependencies, GlobAL finds 7. It misses the dependence between Yahoo! and MSN: even though local detection detects it, global detection removes it because the common data are covered by those between Yahoo! and USWX. This decision is reasonable because Weather.com, from which $\mathrm{Ya}$ hoo! copies, and USWX might derive data from the same source. GLOBAL also misses the dependence between Climaton and MSN: it finds copyings from other sources to Climaton in local detection but eliminates them in global detection. If Climaton is indeed a copier, then the error is caused by wrong direction decisions in local detection. (3) Finally, GLOBAL has three false positives (including Yahoo! $\rightarrow U S W X$ ). For each of such pairs there is more or less cocopying and the global detection does not eliminate it because of some additional shared data (maybe shared at some crawlings). To summarize, except the false negative on Herald $\rightarrow$ WUnderground, errors are mainly caused by wrong direction decisions (because of the lack-of-evidence issue) and uneliminated co-copying (because of the updating issue).

Tbl. 4 compares the results of various methods. First, LOCAL obtains a higher recall (it detects in addition the dependence between Yahoo! and MSN) but a much lower precision. It returns 38 copyings, among which $38-12=26$ are false positives, and GLOBAL removes $26-3=23(88.5 \%)$ of them. Second, BASIC, in contrast, finds only 2 correct copyings. It misses a lot of copyings as it ignores evidence from per-object copying (as illustrated in Ex.3.2 and 


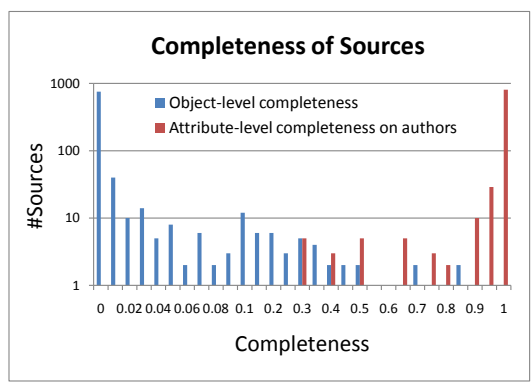

Figure 2: Coverage of AbeBooks sources. 92\% of the sources provide less than $5 \%$ of the books.

\section{ks.}

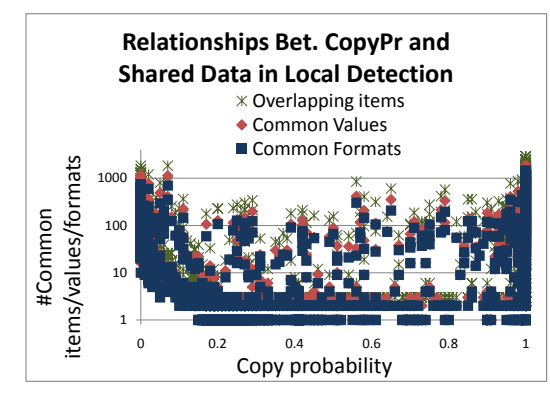

Figure 3: Copy probability vs. shared items, values, formats.
Ex.4.1); in addition, it tends to set $s$ (selectivity) to a high value. Third, ACCU finds 2 golden dependencies (in the correct direction) and 4 silver dependencies. It finds more copyings than BASIC because it considers each shared uncommon value as a wrong value and so accumulates more positive evidence. It has 6 false positives, all co-copyings and transitive copyings. Note, however, that experiments on the synthetic data show improvement of BASIC over ACCU when the true/false notion does apply and there is extra evidence from completeness and formatting of data.

Finally, on average GLOBAL spent 8 seconds for initialization (finding overlapping items, shared values and formats), $2.7 \mathrm{~min}$ utes for local detection, and 10 minutes for global detection (5.9 minutes for finding $\mathbf{R}$ and 4.1 minutes for globally adjusting probabilities). Thus, the efficiency of our algorithm is acceptable when the number of sources is small.

\subsection{Results on AbeBooks data}

Data: The AbeBooks data set was extracted in 2007 from AbeBooks.com by searching computer-science books. In the data set there are 877 bookstores, 1263 books, and 24364 listings, each containing attributes ISBN (key), name, and often authors. Unlike the weather data set, the true/false notion does apply and there is much higher variety in formatting and completeness (Fig.1-2).

Results LoCAL finds 1553 pairs of sources with copying. Fig.3 plots the copying probabilities for pairs whose Jaccard distance on data items (intersection over union) is at least .1. The plot is fairly semetric from left to right, showing that the numbers of shared items, values, and formats themselves do not decide the copying relationship, and our model considers in addition the popularity. GLOBAL finds only 465 pairs with direct copying, as it eliminates co-copying and transitive copying. Compared with LOCAL, it finds that most sources copy from or are being copied by only a few sources (the max is 17 and 9 for GLOBAL respectively, but 44 and 37 for LOCAL).

On average, GLOBAL took 1.6 minutes for initialization, 3.8 minutes for local detection, and 251.1 minutes for global detection. As this data set contains a lot of sources, global detection becomes the bottleneck; especially, between finding $\mathbf{R}$ and globally adjusting probabilities, the former is much more expensive (221.2 min). Fig.4 compares various approximations, including whether to apply Eq.(16) on only "critical" sources (CRISRC), on only "critical" objects (CRIOBJ), and to use $s_{o b j}$ instead of $P\left(S_{1} \stackrel{\text { O.A }}{\longrightarrow} S\right.$ ) (DFTSEL) (Appendix C). We observe that CRISRC considers only critical sources (59\%) and reduced time in finding $\mathbf{R}$, CRIOBJ considers only critical objects (6\%) and reduced time in both steps, and DFTSEL simplifies computation of global probability and also reduced time in both steps. Finally, CRISRCOBJ_DFTSEL took only 26.8 minutes, fairly acceptable given that dependence detection is offline.

\section{RELATED WORK AND CONCLUSIONS}

This paper studied copying detection between a set of sources. We first improved previous techniques for pairwise detection by proposing a framework where we can plug in different types of copying evidence, and consider correlations between copying. We then described techniques for global detection where we eliminate co-copying and transitive copying. Experimental results show high effectiveness and efficiency of our algorithms. Interesting directions for future work include visualization of copying, and categorization and summarization of the copied instances.

Existing work on copying detection includes detecting copying between texts or programs [12, 3], between videos [11], and between structured data sources $[2,6,7]$. The work most related to ours is [6], with which we have compared in detail in Sec.3 and in experiments. Blanco et al. [2] extended [6] by considering multiple attributes, but assumed a copier must copy all attribute values of an object, whereas we consider both per-object (allowing copying only a subset of attribute values) and per-attribute copying.

\section{REFERENCES}

[1] L. Berti-Equille, A. D. Sarma, X. L. Dong, A. Marian, and D. Srivastava. Sailing the information ocean with awareness of currents: Discovery and application of source dependence. In CIDR, 2009.

[2] L. Blanco, V. Crescenzi, P. Merialdo, and P. Papotti. Probabilistic models to reconcile complex data from inaccurate data sources. In CAiSE, 2010.

[3] P. Buneman. The recovery of trees from measures of dissimilarity. Mathematics the Archeological and Historical Sciences, pages 387-395, 1971.

[4] T. M. Cover and J. A. Thomas. Elements of Information Theory. John Wiley \& Sons, Inc., New York, 1991.

[5] X. L. Dong, L. Berti-Equille, Y. Hu, and D. Srivastava. Global detection of complex copying relationships between sources. PVLDB, 2010.

[6] X. L. Dong, L. Berti-Equille, and D. Srivastava. Integrating conflicting data: the role of source dependence. PVLDB, 2(1), 2009.

[7] X. L. Dong, L. Berti-Equille, and D. Srivastava. Truth discovery and copying detection in a dynamic world. PVLDB, 2(1), 2009.

[8] A. K. Elmagarmid, P. G. Ipeirotis, and V. S. Verykios. Duplicate record detection: A survey. IEEE Trans. Knowl. Data Eng., 19(1):1-16, 2007.

[9] E. Gansner, Y. Hu, and S. Kobourov. GMap: Drawing graphs and clusters as map. In IEEE Pacific Visualization Symposium, 2010.

[10] N. Koudas, S. Sarawagi, and D. Srivastava. Record linkage: similarity measures and algorithms. In SIGMOD, 2006.

[11] J. Law-To, L. Chen, A. Joly, I. Laptev, O. Buisson, V. Gouet-Brunet, N. Boujemaa, and F. Stentiford. Video copy detection: a comparative study. In CIVR, pages 371-378, 2007.

[12] S. Schleimer, D. S. Wilkerson, and A. Aiken. Winnowing: Local algorithms for document fingerprinting. In Proc. of SIGMOD, 2003.

[13] X. Yin, J. Han, and P. S. Yu. Truth discovery with multiple conflicting information providers on the web. In Proc. of SIGKDD, 2007. 


\section{APPENDIX}

\section{A. DETAILS FOR THE BASIC MODEL}

We now give details for the basic model. Consider two sources $S_{1}, S_{2} \in \mathcal{S}, S_{1} \neq S_{2}$. As we assume no mutual copying, there are three possible relationships between them: $S_{1}$ copying from $S_{2}$ $\left(S_{1} \rightarrow S_{2}\right), S_{2}$ copying from $S_{1}\left(S_{2} \rightarrow S_{1}\right)$, and neither source copying from the other $\left(S_{1} \perp S_{2}\right)$. We can compute the probability for each case (they sum up to 1) by Bayesian analysis based on our observations of the data, denoted by $\Phi$ :

$$
\begin{aligned}
& P\left(S_{1} \rightarrow S_{2} \mid \Phi\right) \\
= & \frac{\alpha P\left(\Phi \mid S_{1} \rightarrow S_{2}\right)}{\alpha P\left(\Phi \mid S_{1} \rightarrow S_{2}\right)+\alpha P\left(\Phi \mid S_{2} \rightarrow S_{1}\right)+(1-2 \alpha) P\left(\Phi \mid S_{1} \perp S_{2}\right)}{ }^{(19)}
\end{aligned}
$$

Here, $0<\alpha<.5$ is the a-priori probability that a source copies from another. Thus, we need to compute the probability of $\Phi$ conditioned on different copying relationships.

Observation $\Phi$ consists of observations on each data item; i.e., $\Phi=\left\{\Phi_{O . A} \mid O \in \mathcal{O}, A \in \mathcal{A}\right\}$. According to the item-wise independence assumption, we have

$$
P(\Phi \mid \text { cond })=\Pi_{O \in \mathcal{O}, A \in \mathcal{A}} P\left(\Phi_{O . A} \mid \text { cond }\right) .
$$

In local detection, we consider only data provided by $S_{1}$ and $S_{2}$; i.e., $\Phi_{O . A}=\left\{\Phi_{O . A}\left(S_{1}\right), \Phi_{O . A}\left(S_{2}\right)\right\}$. Then, we have (similar for the condition $S_{2} \rightarrow S_{1}$ )

$$
\begin{aligned}
& P\left(\Phi_{O . A} \mid S_{1} \perp S_{2}\right) \\
= & P\left(\Phi_{O . A}\left(S_{1}\right) \mid S_{1} \not S_{2}\right) P\left(\Phi_{O . A}\left(S_{2}\right) \mid S_{2} \not S_{1}\right) \\
& P\left(\Phi_{O . A} \mid S_{1} \rightarrow S_{2}\right) \\
= & P\left(\Phi_{O . A}\left(S_{1}\right) \mid S_{1} \rightarrow S_{2}, \Phi_{O . A}\left(S_{2}\right)\right) P\left(\Phi_{O . A}\left(S_{2}\right) \mid S_{2} \nrightarrow S_{1}\right)(22)
\end{aligned}
$$

Thus, the key to detecting copying is to compute $P\left(\Phi_{O . A}\left(S_{1}\right) \mid\right.$ $\left.S_{1} \nrightarrow S_{2}\right)$ and $P\left(\Phi_{O . A}\left(S_{1}\right) \mid S_{1} \rightarrow S_{2}, \Phi_{O . A}\left(S_{2}\right)\right)$, corresponding to $P\left(\Phi_{O . A}\left(S_{1}\right)\right)$ and $P\left(\Phi_{O . A}\left(S_{1}\right) \mid S_{1} \rightarrow S_{2}\right)$ respectively (similar for $S_{2}$ ). Sec.3 has described their computation according to the data profiling. We now present details in two aspects.

Details for computing $P\left(\Phi_{O . A}\left(S_{1}\right)\right)$ : Here, we need to compute $P\left(\Phi_{O}\left(S_{1}\right) \neq \emptyset\right), P\left(\Phi_{O . A}\left(S_{1}\right) \neq \emptyset\right), P\left(\right.$ value $\left.\left(\Phi_{O . A}\left(S_{1}\right)\right)=v\right)$, and $P\left(\operatorname{format}\left(\Phi_{O . A}\left(S_{1}\right)\right)=f\right)$. Sec. 3 has described how we compute $P\left(\Phi_{O}\left(S_{1}\right) \neq \emptyset\right)$. Similarly we can compute $P\left(\Phi_{O . A}\left(S_{1}\right)\right.$ $\neq \emptyset$ ) according to attribute-level completeness and $P$ (format $\left.\left(\Phi_{O . A}\left(S_{1}\right)\right)=f\right)$ according to format patterns.

Now consider the probability of providing a particular value $v$ (i.e., $P\left(\right.$ value $\left.\left(\Phi_{O . A}\left(S_{1}\right)\right)=v\right)$ ). If $A$ is the key, the probability is 1 . Otherwise, assume there are $m$ wrong values in the underlying domain. Then, $S_{1}$ provides a true value with probability $A\left(S_{1}\right)$ and a particular wrong value with probability $\frac{1-A\left(S_{1}\right)}{m}$ (we assume equal probability of providing a wrong value and relaxation of this assumption is discussed in [6]). Recall that $P(v)$ denotes the probability of value $v$ being true, so

$$
P\left(\operatorname{value}\left(\Phi_{O . A}\left(S_{1}\right)\right)=v\right)=P(v) A\left(S_{1}\right)+(1-P(v)) \frac{1-A\left(S_{1}\right)}{m} \text {. }
$$

Distinguishing negative correlation: Recall that our goal is to find copiers rather than negative correlation, we shall omit evidence for the latter. There are two types of such evidence. First, $S_{1}$ and $S_{2}$ providing the same value in the same format but $P\left(\Phi_{O . A}\left(S_{1}\right) \mid S_{1} \rightarrow\right.$ $\left.S_{2}\right)<P\left(\Phi_{O . A}\left(S_{1}\right)\right)$ shows that a "dependent" source is less likely to provide the same data and indeed implies negative correlation; we set $P\left(\Phi_{O . A}\left(S_{1}\right) \mid S_{1} \rightarrow S_{2}\right)=P\left(\Phi_{O . A}\left(S_{1}\right)\right)$ in this case. Second, $C_{O}\left(S_{1} \mid \neg S_{2}\right)>C_{O}\left(S_{1}\right)$ (similar for $O . A$ ) shows that a "dependent" source is more likely to provide an object not provided by the original source and indeed implies negative correlation; we set $C_{O}\left(S_{1} \mid \neg S_{2}\right)=C_{O}\left(S_{1}\right)$ in this case.

\section{B. PARAMETER SETTING}

The correlated-copying model involves parameters $s_{o b j}, s_{A}, s_{A}^{t}$ and $k$, which can be different for different pairs of sources. We now describe how we set them for each possible copying relationship $S_{1} \rightarrow S_{2}$ (thus, for each pair of sources, we set the parameters differently for the two different directions).

Initialization: We start with $s_{o b j}$. We first examine overlapping objects; for each attribute, we compute the ratio of common values. Accordingly we generate the histogram for each range of ratio, find the peak range, and use its middle value as the selectivity for overlapping objects, $s_{\text {overlap }-o b j}$. Then, the overall selectivity is

$$
s_{\text {obj }}=\frac{s_{\text {overlap-obj }} \cdot\left|\bar{O}\left(S_{1}\right) \cap \bar{O}\left(S_{2}\right)\right|}{\left|\bar{O}\left(S_{2}\right)\right|} .
$$

For each $A \in \mathcal{A}$, we denote by $\overline{O . A}_{v}$ the items on which the two sources provide the same value. Then, we compute $s_{A}$ and $s_{A}^{\rightarrow}$ as

$$
\begin{aligned}
& s_{A}^{\vec{A}}=\frac{\left|\overline{O . A_{v}}\right|}{s_{\text {overlap-obj }} \cdot\left|\bar{O}\left(S_{1}\right) \cap \bar{O}\left(S_{2}\right)\right|} ; \\
& s_{A}^{\rightarrow}=\frac{\left|\overline{O . A}_{v}\right|-s_{A} \cdot s_{\text {overlap }-o b j} \cdot\left|\bar{O}\left(S_{1}\right) \cap \bar{O}\left(S_{2}\right)\right|}{\left(1-s_{\text {overlap }-o b j}\right)\left|\bar{O}\left(S_{1}\right) \cap \bar{O}\left(S_{2}\right)\right|} .
\end{aligned}
$$

Now consider $k$ and we set it for each attribute. Let $\overline{O . A}_{f}$ be the items of $A$ on which the two sources provide the same value in the same format. For the copied objects, the format keeping rate is $\frac{\left|\overline{O . A}_{f}\right|}{\left|\overline{O . A}_{v}\right|}$; for the rest of the objects, we use a default rate $k_{0}$. So

$$
\begin{aligned}
& s_{A}=s_{\text {overlap-obj }} \cdot s_{A}+\left(1-s_{\text {overlap-obj }}\right) s_{A}^{\rightarrow} ; \\
& k_{A}=s_{A} \cdot \frac{\left|\overline{O . A}_{f}\right|}{\left|\overline{O \cdot A}_{v}\right|}+\left(1-s_{A}\right) \cdot k_{0} .
\end{aligned}
$$

Finally, note that we want to avoid extreme values for the parameters and so set them only in a certain range. In our experiments we use range $[.1, .9]$ and truncate values outside this range.

Adjustment: According to our copying detection results, we can adjust the parameters and re-do the detection. In particular, if $S_{1}$ copies from $S_{2}$ (with probability $P\left(S_{1} \rightarrow S_{2}\right)$ ), we shall use the percentage of copied objects or data items (or preserved formatting) observed from the data; otherwise, we shall use the initial settings. Specifically, we adjust the parameters according to the following equations (similar for $s_{\text {overlap-obj }}^{\prime}$ and $s_{A}^{\not^{\prime}}$ ):

$$
\begin{aligned}
s_{o b j}^{\prime}= & \frac{\sum_{O \in \bar{O}\left(S_{2}\right)} s(O)}{\left|\bar{O}\left(S_{2}\right)\right|} P\left(S_{1} \rightarrow S_{2}\right)+s_{o b j}\left(1-P\left(S_{1} \rightarrow S_{2}\right) \chi 29\right) \\
\vec{s}_{A}^{\prime}= & \frac{\sum_{O \in \bar{O}\left(S_{1}\right) \cap \bar{O}\left(S_{2}\right)} s(O) P\left(S_{1} \stackrel{O . A}{\rightarrow} S_{2} \mid \Phi_{O . A}, S_{1} \stackrel{O}{\rightarrow} S_{2}\right)}{\sum_{O \in \bar{O}\left(S_{1}\right) \cap \bar{O}\left(S_{2}\right)} s(O)} \\
& \cdot P\left(S_{1} \rightarrow S_{2}\right)+s_{A}\left(1-P\left(S_{1} \rightarrow S_{2}\right)\right) ; \\
k_{A}^{\prime}= & \frac{\sum_{O . A \in \overline{O . A}_{f}} P\left(S_{1} \stackrel{O . A}{\rightarrow} S_{2} \mid \Phi_{O . A}, S_{1} \rightarrow S_{2}\right)}{\sum_{O \in \bar{O}\left(S_{1}\right) \cap \bar{O}\left(S_{2}\right)} P\left(S_{1} \stackrel{O . A}{\rightarrow} S_{2} \mid \Phi_{O . A}, S_{1} \rightarrow S_{2}\right)} \\
& \cdot P\left(S_{1} \rightarrow S_{2}\right)+k\left(1-P\left(S_{1} \rightarrow S_{2}\right)\right) .
\end{aligned}
$$

Here, $P\left(S_{1} \stackrel{\text { O.A }}{\longrightarrow} S_{2} \mid \Phi_{O . A}, S_{1} \rightarrow S_{2}\right)$ denotes the probability that a copier copies a data item O.A. We compute it in a similar way as we compute $s(O)$ :

$$
\begin{aligned}
& P\left(S_{1} \stackrel{\text { O.A }}{\longrightarrow} S_{2} \mid \Phi_{O . A}, S_{1} \rightarrow S_{2}\right)=s(O) P\left(S_{1} \stackrel{O . A}{\longrightarrow} S_{2} \mid \Phi_{O . A}, S_{1} \stackrel{O}{\longrightarrow} S_{2}\right) \\
& +(1-s(O)) P\left(S_{1} \stackrel{\text { O.A }}{\longrightarrow} S_{2} \mid \Phi_{O . A}, S_{1} \stackrel{O}{\longrightarrow} S_{2}, S_{1} \rightarrow S_{2}\right) ; \\
& P\left(S_{1} \stackrel{\text { O.A }}{\longrightarrow} S_{2} \mid \Phi_{O . A}, S_{1} \stackrel{O}{\longrightarrow} S_{2}\right)=\frac{s_{A}^{\rightarrow} P\left(\Phi_{O . A}\left(S_{1}\right) \mid S_{1} \stackrel{O . A}{\rightarrow} S_{2}\right)}{P\left(\Phi_{O . A}\left(S_{1}\right) \mid S_{1} \stackrel{O}{\longrightarrow} S_{2}\right)} \\
& =\frac{P\left(\Phi_{O . A}\left(S_{1}\right) \mid S_{1} \stackrel{O}{\longrightarrow} S_{2}\right)-\left(1-s_{A}^{\vec{A}}\right) P^{c}\left(\Phi_{O . A}\left(S_{1}\right)\right)}{P\left(\Phi_{O . A}\left(S_{1}\right) \mid S_{1} \stackrel{O}{\rightarrow} S_{2}\right)} .
\end{aligned}
$$




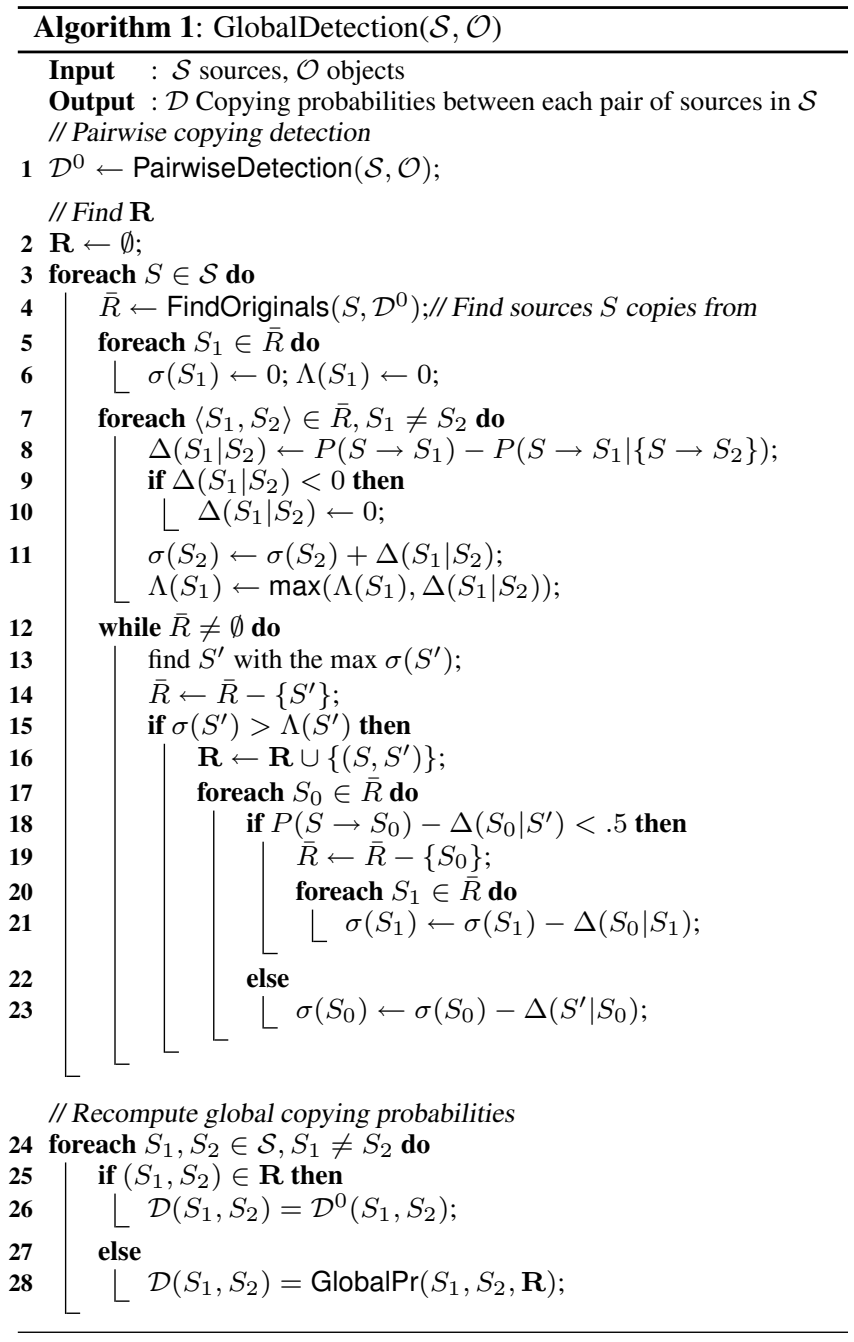

Discussions: Experimental results show that (1) using $s_{\text {overlap-obj }}$ for overlapping objects and $s_{o b j}$ for the rest of the objects obtains better results than using $s_{o b j}$ everywhere; (2) setting $s_{A}, s_{A}^{\rightarrow}$ and $k$ differently for different attributes obtains better results; and (3) setting the parameters empirically can significantly improve over setting arbitrary values, but adjusting the parameters does not show much further benefit.

\section{DETAILS FOR GLOBAL DETECTION}

Algorithm GLOBALDETECTION shows the detailed algorithm for global copying detection. We can further simplify in three ways.

1. When we generate $\mathbf{R}$, we can include in $\bar{R}(S)$ only sources that are likely to cause significant changes or be significantly changed; in particular, those that share a lot of common values with $S$ (not only a high copying probability). In this way, we can reduce $m$ (see Proposition 5.9). (In experiments we consider sources with which $S$ shares $20 \%$ of its values.)

2. When we compute $P\left(S_{1} \rightarrow S_{2} \mid \mathbf{R}\right)$, we can compute $P\left(\Phi_{O . A}\right.$ $\left.\left(S_{1}\right) \mid \mathbf{R}\right)$ only on objects that are strong indicators of copying. Specifically, we start with computing $P\left(\Phi_{O . A}\left(S_{1}\right)\right)$; then, only if $\frac{P\left(\Phi_{O . A}\left(S_{1}\right) \mid S_{1} \rightarrow S_{2}\right)}{P\left(\Phi_{O . A}\left(S_{1}\right) \mid S_{1} \nrightarrow S_{2}\right)}>\tau$ or $\frac{P\left(\Phi_{O}\left(S_{1}\right) \mid S_{1} \rightarrow S_{2}\right)}{P\left(\Phi_{O}\left(S_{1}\right) \mid S_{1} \nrightarrow S_{2}\right)}>\tau$, we compute $P\left(\Phi_{O . A}\left(S_{1}\right) \mid \mathbf{R}\right)$ for each attribute of $O$. Here, $\tau$ is a threshold indicating strong evidence for copying and controls the level of approximation (in experiments we set $\tau=$ $2)$. In this way, we usually need to compute $P\left(\Phi_{O . A}\left(S_{1}\right) \mid \mathbf{R}\right)$ for significantly less data items, but where we do compute $P\left(\Phi_{O . A}\left(S_{1}\right) \mid \mathbf{R}\right)$, we also need to compute $P\left(\Phi_{O . A}\left(S_{1}\right)\right)$.

3. When we apply Eq.(16), instead of using $P\left(S_{1} \stackrel{\text { O.A }}{\longrightarrow} S\right)$, we can use the default selectivity $s_{o b j} s_{A}+\left(1-s_{o b j}\right) s_{A}^{\not}$. In this way, computing $P\left(\Phi_{O . A}\left(S_{1}\right) \mid \mathbf{R}\right)$ is much cheaper.

\section{GOLDEN STANDARD ON WEATHER DATA}

Table 5: Generation of the semi-golden standard for the weather data.

\begin{tabular}{|c|c|l|}
\hline & Rel & \multicolumn{1}{|c|}{ Reason } \\
\hline G & $(1)$ & UniSys lists www.nws.noaa.gov (a mirror of Weather.gov) \\
O & & as a resource. \\
L & $(2)$ & USWX links to Weather.gov in source code. \\
D & $(3)$ & Herald's source code has icons, links from WUnderground. \\
E & $(4)$ & AccuWeather lists CNN as a client. \\
N & $(5)$ & AccuWeather lists WashingtonPost as a client. \\
\hline R & $(6)$ & WeatherBug lists FoxNews as a partner, but shares only \\
E & & 11.4\% of the non-key data. \\
M & $(7)$ & WUnderground lists www.nws.noaa.gov (Weather.gov) but \\
O & & shares only 2 non-key attributes and 16.5\% non-key data. \\
V & $(8)$ & WeatherForYou lists www.nws.noaa.gov (Weather.gov) but \\
E & & shares only 32\% non-key data among attributes and objects. \\
\hline & $(9)$ & Weather.com lists AOL and Yahoo! as affiliates. \\
S & $(10)$ & Weather.com lists MSN and Yahoo! as affiliates. \\
I & $(11)$ & WDT lists CNN and FoxNews as customers. \\
L & $(12)$ & Potential co-copiers and share a lot of data. \\
V & $(13)$ & Potential co-copiers and share a lot of data. \\
\cline { 2 - 3 } E & $(14)$ & No explicit claim from Herald but share a lot of data. \\
R & $(15)$ & No explicit claim from FindLocalWeather but share a lot of data. \\
& $(16)$ & No explicit claim from Climaton but share a lot of data. \\
& $(17)$ & No explicit claim from NYTimes but share a lot of data. \\
\hline
\end{tabular}

\section{E. EXPERIMENTS ON SYNTHETIC DATA}

To test effectiveness of our models, we also experimented on synthetic data. To generate a copier $C$, we chose an original source $S$ and a copier template $T$ from the AbeBooks data set. Copier $C$ copies from $S$ and independently provides some values or uses some formats, for which we use those provided by $T$; essentially $C$ is a copier of both $S$ and $T$, but we discarded $T$. We assume $C$ provides data in three steps: (1) among books in $\bar{O}(S) \cap \bar{O}(T)$, $C$ copies $p_{o}$ percent on all attribute values from $S$, then for each attribute $A$, modifies $m_{A}$ percent of the copied values (per-object copying); (2) for the rest of the books in $\bar{O}(S) \cap \bar{O}(T)$, for $p_{a}$ percent of non-key attributes $C$ copies all values from $S$, then modifies $m_{A}^{\prime}$ percent of the values for each $A$ (per-attribute copying); (3) for other data items in $\bar{O}(T), C$ provides values on its own while copies $c_{A}$ percent for each $A$. For $p_{f}$ percent of the copied items, $C$ keeps the copied format and for the rest it changes the format. We believe real-world copiers copy in a more or less similar fashion, though may skip some steps or change their order. We randomly decided which data to copy and modify, and generated $m_{A}, m_{A}^{\prime}$ and $c_{A}$ between $[0,2 m]$ by Gaussian distribution with mean $m$. By default we set $p_{o}=.8, p_{a}=0, p_{f}=1$, and $m=.1$.

We considered three cases: (1) transitive copying: five copiers $C_{1}-C_{5}$, where $C_{1}$ copies from $S$ and $C_{i+1}$ copies from $C_{i}, i \in$ $[1,4]$; (2) co-copying: five copiers all copying from $S$; (3) multicopying: one copier copying sequentially from $S_{1}-S_{5}$ (i.e., copying from $S_{i}$ a random subset of $\bar{O}\left(S_{i}\right) \cap \bar{O}(T)-\cup_{j=1}^{i-1} \bar{O}\left(S_{j}\right), i \in$ $[1,5]$ ). We pre-selected 10 sources $\bar{S}$ whose object-level completeness ranges from 0.05 to .9 (not necessarily independent). For (1) and (2), for each $S \in \bar{S}$ we ran the experiments 10 times; at each time we randomly chose 5 templates that are judged as independent of $S$ by local detection (but not necessarily independent be- 


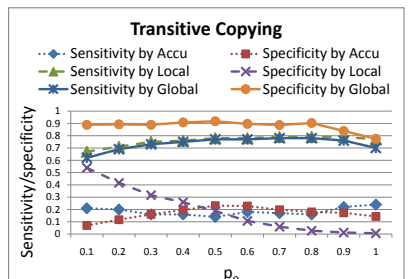

Figure 6: Transitive copying.

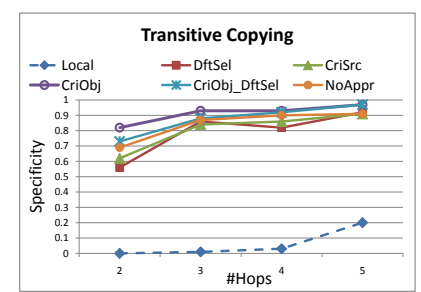

Figure 7: Specificity vs. \#hops.

tween themselves) and differ in completeness by at least .05. For (3), we ran the experiment 100 times, each time randomly choosing a sequence of 5 sources from $\bar{S}$ and trying to ${ }^{8}$ randomly choose 10 templates that are independent of them and differ in completeness by at least .05; we detected copying for each copier separately. For all cases, we reported the average results.

We set parameters by (1) setting default values (FIXPARA) where $s_{o b j}=s=k=.8, s_{A}=.9, s_{A}^{\nrightarrow}=.4 ;$ (2) setting empirical values (EMPPARA); and (3) first setting empirical values and then adjusting them (ADJPARA) (Appendix B). By default, we applied EMPPARA. We used true values decided from the real-world data, setting $p(v)=1$ for true values and $p(v)=0$ for false ones.

To better quantify how we detect transitive and co-copying, we report sensitivity, the proportion of real copying that are identified with the correct direction (a strict version of recall), and specificity, the proportion of non-copyings that are identified as such.

Transitive copying: We varied $p_{o}$ from .1 to 1 and examined copying between sources in $\left\{S, C_{1}, \ldots, C_{5}\right\}$ (Fig.6). We observe that while GLOBAL slightly reduces sensitivity (by $3 \%$ ) compared with LOCAL, it significantly improves specificity (avg .88). Also, GLOBAL obtains fairly stable results when $p_{o}$ varies: when $p_{o}$ is very small, the sensitivity is slightly lower because a copier can copy very few data and is not detectable; when $p_{o}$ is very high, the sensitivity and specificity are slightly lower because a copier may transitively copy a lot of data from its transitive ancestor and even share some additional data (local detection found an average copying probability of .39 between templates), which is indistinguishable from direct copying. Finally, ACCU (hereafter we set $s=p_{o}(1-m)$ ) performs worst; it assumes item-wise independence and considers only accuracy, so can often make mistakes about copying direction (it finds all direct copying but only $18 \%$ in the correct direction).

Table 6 compares various approximation methods and Fig. 7 gives more details on specificity w.r.t. \#hops between sources (\#hops between $C_{i}$ and $C_{j}, i>j$, is $i-j$ and \#hops between $C_{i}$ and $S$ is $i$; 1-hop indicates direct copying) when $s_{o}=.8$. With no surprise, the more hops, the higher specificity; when \#hops $>2$, CRIOBJ obtains a specificity of above .9. Among the approximations, CRIOBJ spends half of the execution time as NOAPPR but obtains the best results (as it is not biased by effects on non-critical objects). CRIOBJ_DEFSEL further cuts the execution time by $70 \%$ but still obtains better results than NOAPPR; however, it reduces the specificity by $6 \%$ compared with CRIOBJ.

Co-copying: Again, we varied $p_{o}$ and examined copying between $\left\{S, C_{1}, \ldots, C_{5}\right\}$ (Fig.8). GLOBAL again reduces sensitivity slightly (by $10 \%$ ) and improves specificity significantly. However, we observe two differences from transitive copying. First, specificity

\footnotetext{
${ }^{8}$ Sometimes this is impossible when there are insufficient number of sources independent of $S_{1}-S_{5}$.
}

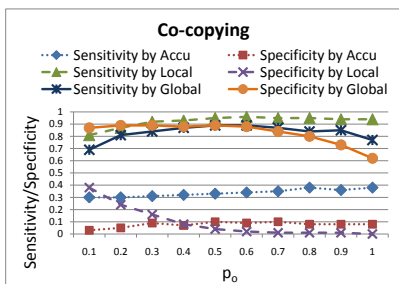

Figure 8: Co-copying.

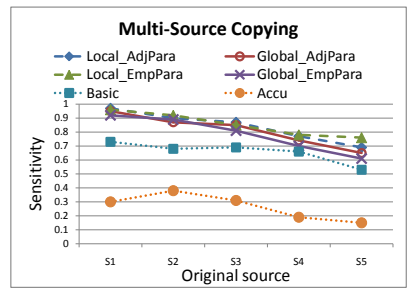

Figure 9: Multi-source copying.
Table 6: Approximation for detecting transitive copying.

\begin{tabular}{|c|c|c|c|c|c|}
\hline & NOAPPR & CRISRC & CRIOBJ & DFTSEL & CRIOBJ_DFTSEL \\
\hline Sensitivity & .75 & .75 & .77 & .7 & .78 \\
Specificity & .81 & .76 & .89 & .74 & .84 \\
Time(s) & 99.3 & 81.8 & 50.2 & 32.4 & 14.9 \\
\hline
\end{tabular}

is less stable: when $p_{o}=1$, the specificity is only .62. When $p_{o}$ is high, co-copiers can share a large number of copied values and maybe some additional values, so indistinguishable from direct copying. Second, LOCAL obtains higher sensitivity than in the transitive-copying case (avg .94 vs. .76). Actually, we have detected all copying in both cases, but did worse in finding the correct direction in case of transitive copying, as there the randomly chosen "original" sources are likely to have high completeness and lead to less precise direction detection.

Multi-copying: We examined copying between the copier and each original source (sensitivity) (Fig.9). We have five observations. (1) When a copier really copies from multiple sources, GLOBAL reduces the sensitivity only very slightly (GLOBAL_ADJPARA by $3 \%$ ). (2) The sensitivity reduces from $S_{1}$ to $S_{5}$, as the copier tends to copy less (if any) data from sources towards the end of the sequence. (3) ADJPARA does not necessarily improve over EMPPARA for LOCAL, but by using it GLOBAL reduces the sensitivity much less (avg 3\% vs. 8\%) (however, GLOBAL can obtain lower specificity using ADJPARA in case of co-copying and transitive copying; details skipped). (4) BASIC does not consider object copying so obtains much lower sensitivity; (5) ACCU considers only accuracy and uses the same selectivity for all source pairs, so performs the worst.

Finally, we observed similar results for per-attribute copying $\left(p_{o}=0, p_{a}=.5\right)$, and combined copying $\left(p_{o}=.5, p_{a}=.5\right)$, and fairly stable results when we varied $p_{o}, p_{f}, m_{a}$ and $p_{a}$.

Summary: We summarize our experimental results as follows.

- Global improves over LOCAL significantly on identifying complex copying relationships.

- Among various approximations for global detection, CRIOBJ can cut the execution time by half or more but still obtain the best results. CRIOBJ_DFTSEL further reduces the execution time without sacrificing the results too much.

- In local detection, BASIC improves over ACCU significantly on copying-direction judgment by considering completeness and formatting in addition to accuracy, using both sourcewise and data-item-wise measures, and using $P^{c}\left(\Phi_{O . A}\left(S_{1}\right)\right)$ in computation of $P\left(\Phi_{O . A}\left(S_{1}\right) \mid S_{1} \rightarrow S_{2}\right)$. LOCAL improves over BASIC further by considering object copying.

- Setting parameters using EMPPARA beats FIXPARA significantly and can obtain quite stable results. ADJPARA does not show further benefits in either local or global detection. 


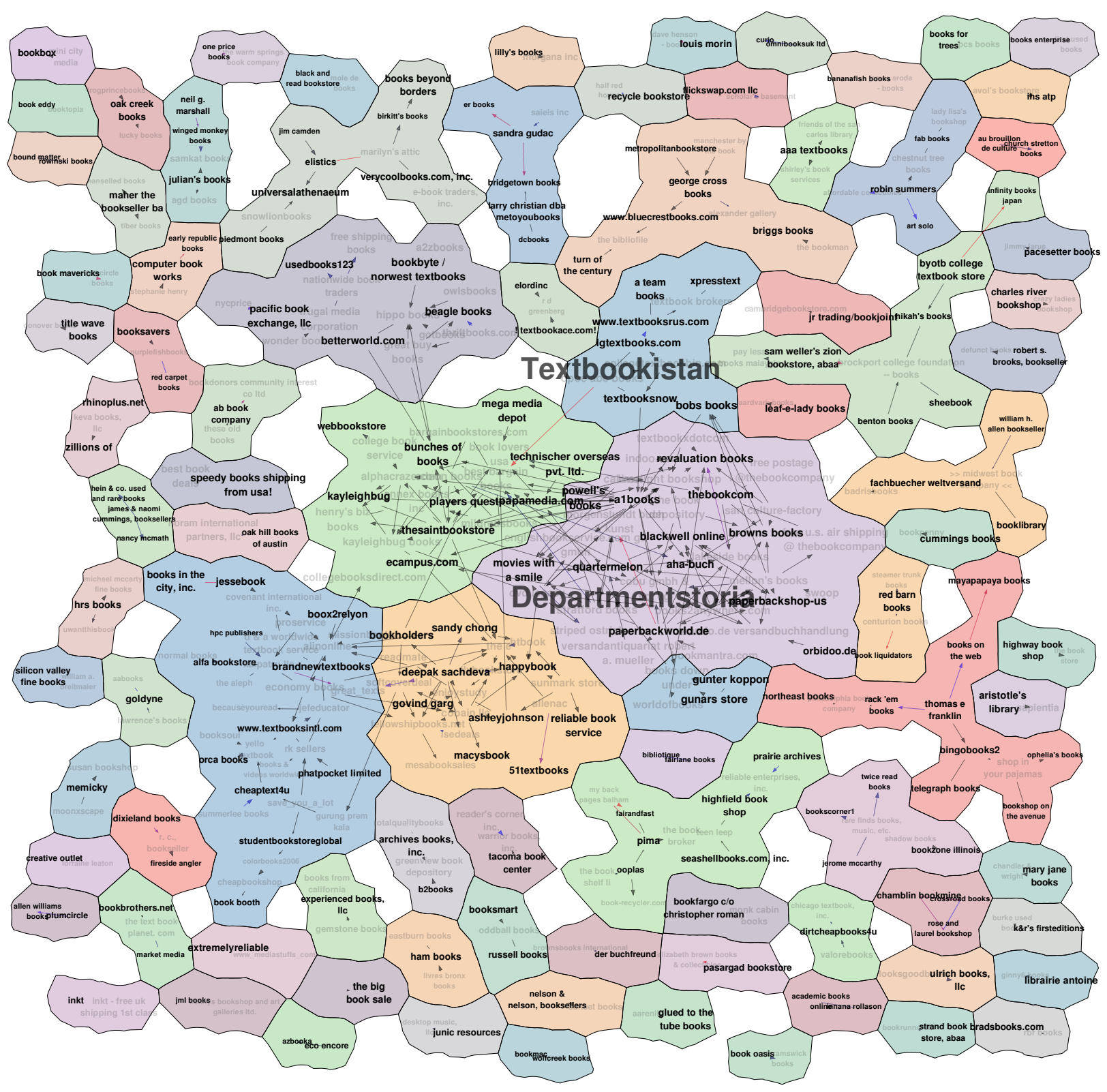

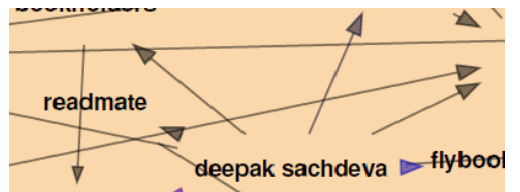

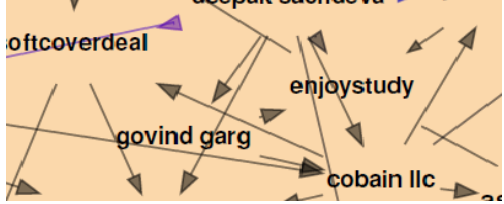

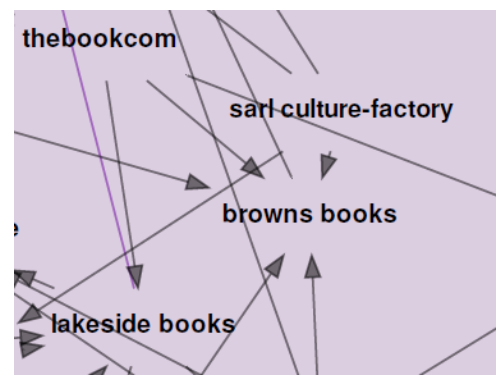

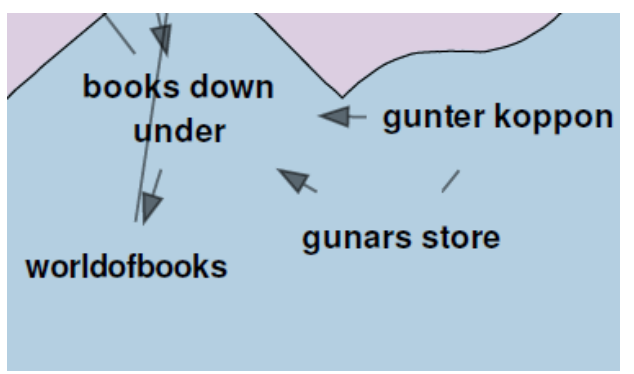

Figure 10: Map of AbeBooks data sources: the copying relationships identified by our copying-detection model. Each "node" represents a data source and the size of the font corresponds to the number of provided books; to avoid cluttering, we show overlapping ones half transparently. An edge $S_{1} \rightarrow S_{2}$ indicates that $S_{1}$ copies from $S_{2}$; the size of the arrow indicates our confidence of the copying direction; the color indicates the probability of copying (black for 1 , blue for .75 , and red for .5 , and oftergogbabilities are represented by a blend of these colors; e.g., purple for .5-.75.). Each "country" represents a cluster of sources, clustered by modularity clustering (see "Clauset, A., Newman, M.E.J., Moore, C.: Finding community structure in very large networks. Physical Review E 70, 066111 (2004)") based on their copying relationships. 